\title{
Increased Mitochondrial Mass and Cytosolic Redox Imbalance in Hippocampal Astrocytes of a Mouse Model of Rett Syndrome: Subcellular Changes Revealed by Ratiometric Imaging of JC-1 and roGFP1 Fluorescence
}

\author{
Dörthe F. Bebensee, ${ }^{1,2}$ Karolina Can, ${ }^{1,2}$ and Michael Müller ${ }^{1,2}$ \\ ${ }^{1}$ Center for Nanoscale Microscopy and Molecular Physiology of the Brain (CNMPB), Georg-August-Universität Göttingen, \\ Universitätsmedizin Göttingen, Göttingen, Germany \\ ${ }^{2}$ Zentrum Physiologie und Pathophysiologie, Institut für Neuro- und Sinnesphysiologie, Humboldtallee, 23 Göttingen, Germany \\ Correspondence should be addressed to Michael Müller; mmuelle7@gwdg.de
}

Received 15 March 2017; Revised 16 June 2017; Accepted 27 June 2017; Published 13 August 2017

Academic Editor: Icksoo Lee

Copyright (c) 2017 Dörthe F. Bebensee et al. This is an open access article distributed under the Creative Commons Attribution License, which permits unrestricted use, distribution, and reproduction in any medium, provided the original work is properly cited.

\begin{abstract}
Rett syndrome (RTT) is a neurodevelopmental disorder with mutations in the MECP2 gene. Mostly girls are affected, and an apparently normal development is followed by cognitive impairment, motor dysfunction, epilepsy, and irregular breathing. Various indications suggest mitochondrial dysfunction. In Rett mice, brain ATP levels are reduced, mitochondria are leaking protons, and respiratory complexes are dysregulated. Furthermore, we found in MeCP2-deficient mouse (Mecp $2^{-/ y}$ ) hippocampus an intensified mitochondrial metabolism and ROS generation. We now used emission ratiometric 2-photon imaging to assess mitochondrial morphology, mass, and membrane potential $(\Delta \Psi \mathrm{m})$ in $M e c p 2^{-l y}$ hippocampal astrocytes. Cultured astrocytes were labeled with the $\Delta \Psi \mathrm{m}$ marker JC-1, and semiautomated analyses yielded the number of mitochondria per cell, their morphology, and $\Delta \Psi \mathrm{m} . \mathrm{Mecp}^{-l y}$ astrocytes contained more mitochondria than wild-type (WT) cells and were more oxidized. Mitochondrial size, $\Delta \Psi \mathrm{m}$, and vulnerability to pharmacological challenge did not differ. The antioxidant Trolox opposed the oxidative burden and decreased the mitochondrial mass, thereby dampening the differences among WT and $M e c p 2^{-l y}$ astrocytes; mitochondrial size and $\Delta \Psi \mathrm{m}$ were not markedly affected. In conclusion, mitochondrial alterations and redox imbalance in RTT also involve astrocytes. Mitochondria are more numerous in Mecp $2^{-l y}$ than in WT astrocytes. As this genotypic difference is abolished by Trolox, it seems linked to the oxidative stress in RTT.
\end{abstract}

\section{Introduction}

Rett syndrome (RTT) is a postnatal, X-chromosome linked, progressive neurodevelopmental disorder. The first RTT cases were reported in 1966 by the Austrian pediatrician Andreas Rett [1]; the characteristic symptoms include autistic features, dementia, motor dysfunction, loss of facial expressions, stereotypical hand movements, severe respiratory disturbances, and epilepsy [1-3]. RTT almost exclusively affects girls, typically at an incidence of 1:10,000-15,000 [4]. In boys, it is either lethal or due to severe neonatal encephalopathy death occurs during the first year [5]. Despite being a rare disease, RTT - next to Down syndrome-is the second most common genetic cause of severe cognitive disabilities in girls [4].

The main causes of classical RTT are de novo mutations of the MECP2 (methyl-CpG-binding protein 2) gene. It is located on the long arm of the X chromosome (Xq28) [6] and functions as a transcriptional modulator by either mediating gene silencing [7] or acting as transcriptional activator [8]. The spontaneous $M E C P 2$ mutations underlying RTT mostly occur in the paternal X chromosome $[9,10]$. In the affected girls, the disease manifests in early childhood and progresses in four stages: An initial and apparently normal 
development (stage 1) is followed by a fast regression (stage 2), and then, a plateau or pseudostationary phase manifests (phase 3 ), which is concluded by a late motor deterioration (phase 4) $[3,11]$. The life expectancy varies with clinical severity, and some Rett patients may reach the age of 70 years [12]. Often, death arises from cardiac/respiratory insufficiency, acute infections, or sudden incidents at night $[12,13]$. A causal therapy for RTT is currently not available. Yet, some symptoms can be partly ameliorated by pharmacotherapy $[14,15]$, occupational therapy, and/or physical therapy $[16,17]$.

The past years revealed that RTT is closely associated with mitochondrial alterations, and in view of the multitude of mitochondrial functions, these defects were proposed to contribute to disease progression $[18,19]$. It was even speculated whether RTT may represent a mitochondrial disease [20]. Morphological alterations of mitochondria were first detected in muscle biopsy samples of Rett patients, whose mitochondria appeared swollen or dumbbell-shaped [21] and exhibited granular inclusions, vacuolizations, and membranous changes $[22,23]$. Post mortem studies on frontal lobe confirmed structural changes of mitochondria also for the brain [22].

Detailed follow-up biochemical analyses revealed a reduced expression of a subunit of cytochrome c oxidase (complex IV of the respiratory chain) in post mortem frontal cortex [24], which could impair ATP synthesis. Furthermore, comparative gene-array analyses on patient lymphomonoytes indicated a differential expression of various genes pivotal to mitochondrial function and/or organization [25]. In mouse models of RTT, symptomatic animals also showed reduced enzyme activities of respiratory chain complexes II, III, IV, and ANT1, as well as reduced glutathione levels in the brain and/or skeletal muscle [26-29]. Furthermore, the ANT1 gene encoding the mitochondrial adenine nucleotide translocase is highly upregulated in the $M e c p 2^{-l y}$ mouse brain and Rett patient fibroblasts [30], the latter of which also show clear signs of metabolic mitochondrial dysregulation, oxidative stress, and diminished redox-balancing capabilities [31]. Also, the elevated blood lactate and pyruvate levels suggest defects in the mitochondrial respiratory chain and the urea cycle [32]. Obvious consequence of these mitochondrial changes is a less efficient respiratory chain and thus limited ATP synthesis. Indeed, magnetic resonance tomography and biochemical assays confirmed reduced brain ATP levels in male and female Mecp2mutant mice $[26,33]$. In view of the severe respiratory disturbances and frequent apneas in Rett patients [3436], systemic hypoxia may occur and challenge mitochondrial function further. This led to the assumption that the various mitochondrial abnormalities are causal events in RTT. They may contribute to the complex symptoms of RTT and promote disease progression either directly or indirectly by generating free radicals and provoking redox imbalance $[18,19,28,29,37-40]$.

The mostly neuronal alterations in RTT [41-43] originally suggested a purely neuronal disorder. Only later glial cells were confirmed to contain MeCP2 and to be affected by Mecp2 mutations as well [44]. Meanwhile, it is clear that also glial cells contribute to disease progression by exerting negative effects on neurons $[45,46]$. In MeCP2-deficient mice, re-expression of MeCP2 specifically in astrocytes improved motor function, respiratory regularity, and anxiety, and the life-expectancy increased significantly as compared to mice lacking MeCP2 [45]. Restoring MeCP2 in oligodendrocytes was also beneficial, ameliorating motor symptoms and normalizing body weights [46]. Also, MeCP2-deficient microglia contribute to disease progression, as its intensified glutamate release provokes dendritic malformations, microtubule derangement, and damage of postsynaptic glutamatergic components [47]. Therefore, we here assessed to what degree also astrocytes show mitochondrial alterations. Using advanced optical tools, we took a closer look at the function and morphology of these organelles in cultured astrocytes of neonatal $M e c p 2^{-l y}$ and WT mice and also quantified cellular redox conditions. Our particular focus was on the hippocampus, as this brain area is metabolically demanding and vulnerable to oxidative stress.

\section{Materials and Methods}

This study was performed on Mecp2 knockout mice [B6.129P2(C)-Mecp2 $2^{\text {tm.1.1Bird }}$, [48]]. To ensure uniform conditions, that is, total MeCP2 deficiency, only male wild-type (WT) and male Rett mice $\left(M e c p 2^{-l y}\right)$ were used. Mecp $2^{-/ y}$ mice show a more severe disease progression and develop earlier symptoms as compared to heterozygous female Rett mice [48]. All procedures met the German regulations and were authorized by the Office of Animal Welfare of the University Medical Center Göttingen.

2.1. Solutions. If not mentioned differently, all compounds were purchased from Sigma-Aldrich. The artificial cerebrospinal fluid (ACSF) contained $130 \mathrm{mM} \mathrm{NaCl}, 3.5 \mathrm{mM} \mathrm{KCl}$, $1.25 \mathrm{mM} \quad \mathrm{NaH}_{2} \mathrm{PO}_{4}, 24 \mathrm{mM} \quad \mathrm{NaHCO}_{3}, \quad 1.2 \mathrm{mM} \mathrm{CaCl}$, $1.2 \mathrm{mM} \mathrm{MgSO}_{4}$, and $10 \mathrm{mM}$ dextrose. It was aerated continuously with carbogen gas mixture $\left(95 \% \mathrm{O}_{2}, 5 \% \mathrm{CO}_{2}\right)$ to maintain a stable $\mathrm{pH}$ of 7.4 and a proper $\mathrm{O}_{2}$ supply of the cells during the imaging experiments.

Minimum essential cell culture medium (MEM, Invitrogen) was supplemented with $0.2 \mathrm{mg} / \mathrm{ml} \mathrm{NaHCO}_{3}$, $0.1 \mathrm{mg} / \mathrm{ml}$ transferrin (Calbiochem/Merck), and $5 \mathrm{mg} / \mathrm{ml}$ glucose. For the initial plating of the cells, it furthermore contained 10\% FCS (fetal calf serum, Biochrom), $25 \mu \mathrm{g} / \mathrm{ml}$ insulin, and $2 \mathrm{mM}$ L-glutamine. After the first day in culture, a slightly different medium was used (growth medium), which contained 5\% FCS, $0.5 \mathrm{mM}$ L-glutamine, $20 \mu \mathrm{l} / \mathrm{ml}$ B27 50x supplement (Invitrogen), and $100 \mu \mathrm{g} / \mathrm{ml}$ penicillinstreptomycin (Biochrom).

For most drugs, we first prepared stock solutions. Sodium cyanide $\left(\mathrm{CN}^{-}\right)$was dissolved as $1 \mathrm{M}$ aqueous stock and stored at $-20^{\circ} \mathrm{C}$; other experimental solutions containing $\mathrm{CN}^{-}$were prepared from this stock right before use. FCCP (carbonylcyanide-4-(trifluoromethoxy) phenylhydrazone, Tocris Bioscience) was dissolved as $10 \mathrm{mM}$ stock in dimethyl sulfoxide (DMSO) and stored at $4^{\circ} \mathrm{C}$. The mitochondrial markers JC-1 (5,5',6,6'-tetrachloro-1,1',3,3'-tetraethylbenzimidazolylcarbocyanine iodide, Thermo Fisher Scientific) 
and MitoTracker Red FM (Thermo Fisher Scientific) were dissolved in DMSO as $2 \mathrm{mg} / \mathrm{ml}$ and $1 \mu \mathrm{M}$ stocks, respectively, and kept frozen. The free-radical scavenger Trolox (( \pm$)$-6-hydroxy-2,5,7,8-tetramethylchromane-2-carboxylic acid) was first dissolved in DMSO and then added to cell culture medium for overnight treatment.

\subsection{Preparation of Dissociated Hippocampal Cell Cultures.} Cell cultures of hippocampal neurons and glial cells were prepared from 2- to 4-day-old mice as described earlier [49]. In brief, mice were decapitated, the brain was gently but quickly removed and immersed in ice-cold HBSS (Hank's balanced salt solution) containing 20\% FCS. Both hippocampi were then isolated, cut with a scalpel into smaller pieces, and trypsinated $\left(5 \mathrm{mg} / \mathrm{ml}, 10 \mathrm{~min}, 37^{\circ} \mathrm{C}\right)$. The cells were then dissociated by trituration and centrifuged $\left(1500 \mathrm{rpm}, 10 \mathrm{~min}, 4^{\circ} \mathrm{C}\right)$, and the pellet obtained was resuspended. Next, this cell suspension was plated on Matrigel (BD Biosciences)-coated glass cover slips, which were placed in 4-well culture plates (Nunc). Dissociated cell cultures were incubated at $37^{\circ} \mathrm{C}\left(5 \% \mathrm{CO}_{2}\right.$ atmosphere). After $24 \mathrm{~h}$, the medium was replaced with growth medium, and after another 3 days, growth factors as well as half of the medium were refreshed again. Experiments were performed between 3 and 10 days in vitro.

2.3. Multiphoton Imaging of Mitochondrial JC-1 Fluorescence. To visualize mitochondria and monitor their membrane potentials $(\Delta \Psi \mathrm{m})$, the membrane potential probe JC-1 was chosen. JC-1 accumulates in mitochondria depending on their $\Delta \Psi \mathrm{m}$, and it is ratiometric by emission [50-55]. Cells were bulk loaded with JC-1 $(1 \mu \mathrm{g} / \mathrm{ml}, 15 \mathrm{~min})$ immediately before the experiments.

Our 2-photon laser-scanning microscope (TPLSM, Figure 1) is composed of a Ti: Sa laser system (Mai Tai eHP DS, Newport-Spectra Physics) and an upright microscope (BX51WI, Olympus) equipped with a TriM Scope II scanhead and ImSpector Control Software (LaVision BioTec). To separate the emitted fluorescence and the laser excitation, a $670 \mathrm{~nm}$ beam splitter (670DCXXR) was combined with an IR-block filter (HC 680/SP). Fluorescence was detected in nondescanned mode by highly sensitive photomultiplier tubes (PMTs; H7421/H7422, Hamamatsu). For emission ratiometric analyses, JC-1 was excited at $925 \mathrm{~nm}$, and the emission was divided further in its green and red spectral components by using a $570 \mathrm{~nm}$ beam splitter (570DCXR) as well as $525 / 50 \mathrm{~nm}$ (green) and $617 / 73 \mathrm{~nm}$ (red) bandpass filters [54]. All optical filters were obtained from AHF Analysentechnik AG (Tübingen, Germany). For optimized optical resolution and detection efficiency, we chose a $63 \mathrm{x}$ 1.0 NA water immersion objective (Plan Apochromat VISIR, Zeiss).

For the experiments, cell culture cover slips were placed in a submersion-style recording chamber, which was perfused continuously with warm $\left(37^{\circ}\right) \mathrm{ACSF}$ at $4 \mathrm{ml} / \mathrm{min}$ flow rate. JC-1-labeled cells were allowed to adjust for 15-20 min, and then experiments were started. Imaging of individual cells was performed using a scanfield size of $80 \times 80 \mu \mathrm{m}$ (resolution $80 \mathrm{~nm} /$ pixel) and $1200 \mathrm{~Hz}$ line-scanning frequency. To reduce pixel noise, 5-fold line averaging was applied. All 3-dimensional (3-d) image stacks were recorded with axial $(Z)$-increments of $0.25 \mu \mathrm{m}$.

Before the analysis, each 3-d stack underwent blind deconvolution (Autodeblur 9.3, AutoQuant Imaging), based on the objective used (1.0 NA) and the center wavelengths of the two different emission channels $(525 \mathrm{~nm}$ and $617 \mathrm{~nm})$. For all further image analyses, Metamorph Offline 7.5 (Molecular Devices) was used. Background subtraction was not performed during the ratiometric calculations, as in the case of 2-photon excitation, out-of-focus light is virtually nonexisting. However, to ensure a sufficient coverage of the 8 -bit pseudocolor palette, a scaling factor of 200 was introduced, and the JC-1 ratio was calculated as

$$
R_{\mathrm{JC}-1}=\left(\frac{F_{\operatorname{green}(530 \mathrm{~nm})} * 200}{F_{\text {red }(590 \mathrm{~nm})}}\right) .
$$

2.4. Excitation Ratiometric Redox Imaging. Cytosolic redox balance was determined with the excitation ratiometric optical redox indicator roGFP1 (oxidation-reduction sensitive green fluorescent protein 1) [56]. Within 2-3 days upon transfection (lipofection) with roGFP1 expressing plasmids (see [57]), cultured astrocytes sufficiently expressed roGFP1. For excitation-ratiometric analyses, roGFP1 was excited alternately at $395 \mathrm{~nm}(100 \mathrm{~ms}$ exposure) and $470 \mathrm{~nm}(150 \mathrm{~ms}$ exposure) with frame rates of $0.1 \mathrm{~Hz}$. The emission was recorded by a fully computerized CCD-camera imaging system (Polychrome II, TILL Photonics) at $525 \mathrm{~nm}$, using a 63x 1.0 NA objective (Zeiss W-Plan Apochromat VIS-IR) and applying $4 \times 4$ pixel binning. The roGFP1 fluorescence ratio $\mathrm{F}_{395} / \mathrm{F}_{470}$ was calculated in real time, as described earlier [40, 57-59]. For true quantitation, roGFP1 responses were calibrated to saturating doses of $\mathrm{H}_{2} \mathrm{O}_{2}(5 \mathrm{mM}, 5 \mathrm{~min})$ and DTT $(10 \mathrm{mM}, 5 \mathrm{~min})$, to obtain those ratiometric values representing complete sensor oxidation/reduction. Based on these calibrations, the relative degree of roGFP1 oxidation $\left(\mathrm{OxD}_{\text {roGFP1 }}\right)$ and the reduction potential $\left(\mathrm{E}_{\mathrm{roGFP}}\right)$ was calculated $[57,58,60,61]$ for WT and $M e c p 2^{-l y}$ astrocytes.

2.5. MitoTracker-Based Visualization of Mitochondria. Occasionally, we used MitoTracker Red to visualize mitochondria. Bulk-loaded cell cultures $\left(1 \mu \mathrm{g} / \mathrm{mg}, 20 \mathrm{~min}, 37^{\circ} \mathrm{C}\right)$ were excited at $550 \mathrm{~nm}$, using the abovementioned CCD-camera imaging system and a 40x $0.8 \mathrm{NA}$ water immersion objective (Zeiss Achroplan). MitoTracker emission was separated by a $570 \mathrm{~nm}$ dichroic mirror and a $590 \mathrm{~nm}$ longpass emitter, and single images were taken at an exposure time of $300 \mathrm{~ms}$. Pixel binning was not applied.

2.6. Statistics. The analyzed neuron/glial cultures were obtained from 14 male WT and 15 male Mecp $2^{-l y}$ mice. During experiments and data analyses, the genotype was still blinded; genotyping was performed at a later time-point from tail biopsies collected during dissection. Data are shown as mean \pm standard deviation, $n$ reports the number of astrocytes studied. Depending on the type of experiment, significance of the differences and drug effects observed were determined using either paired or unpaired two-tailed 


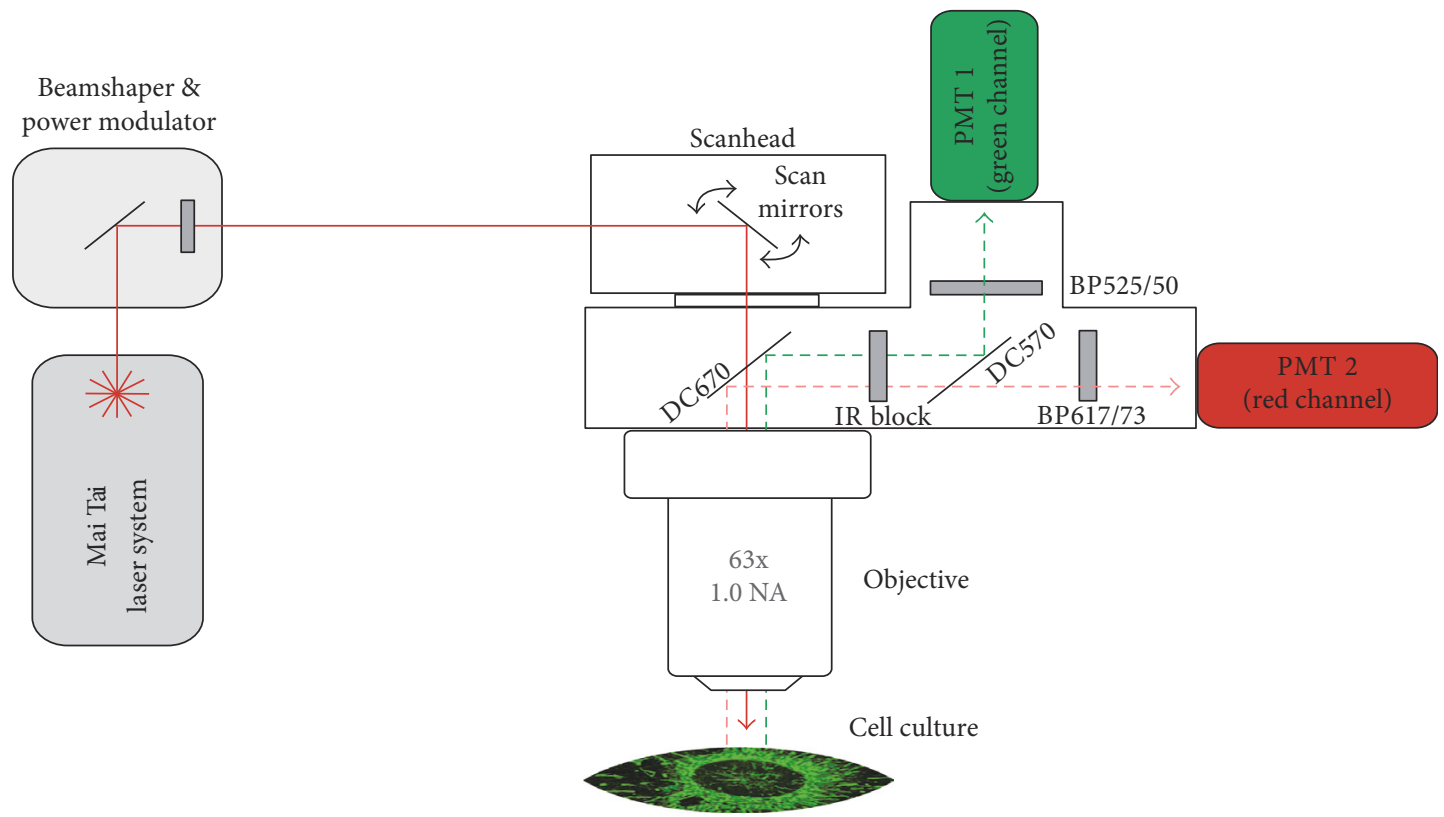

(a)
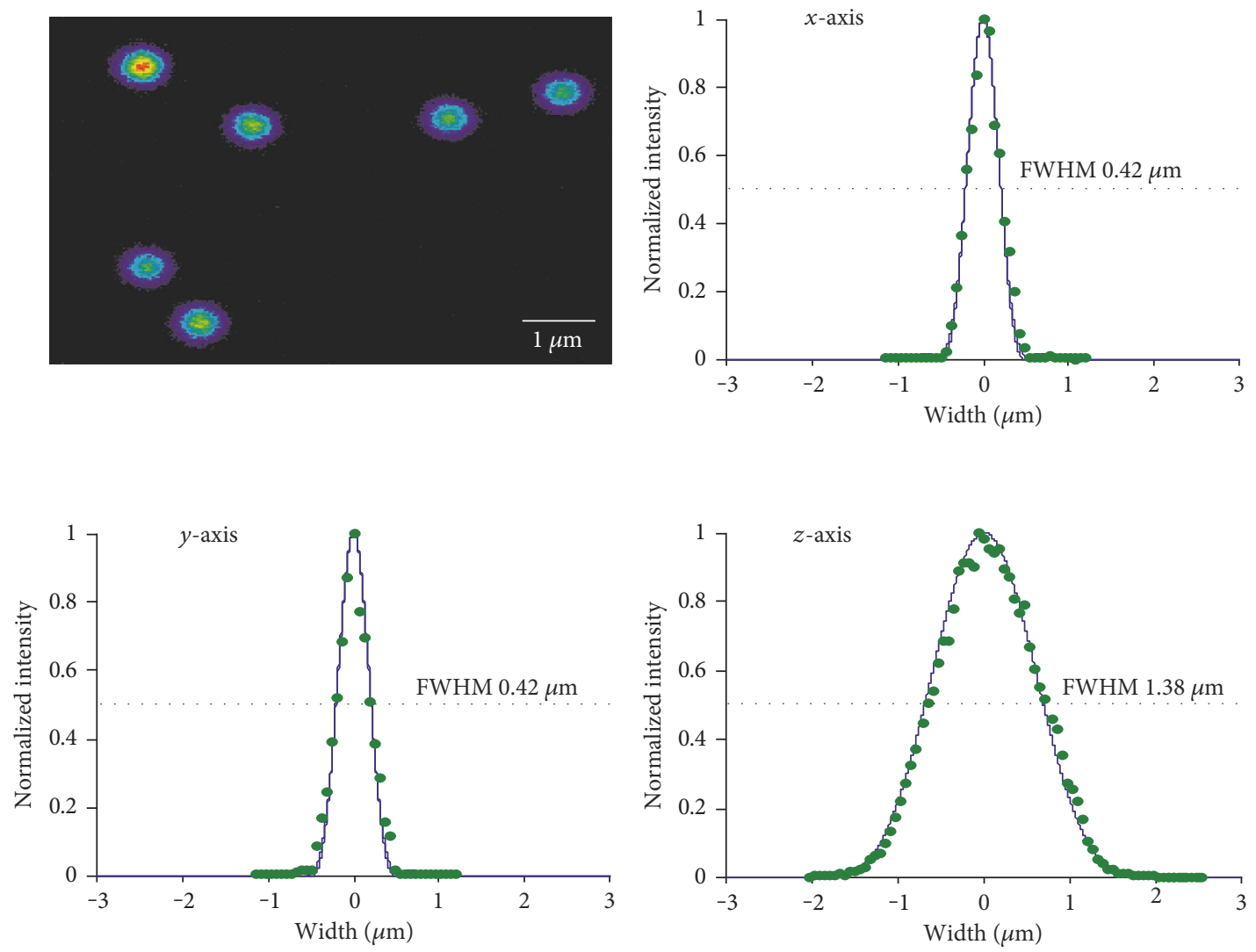

(b)

FIgURE 1: Two-photon laser-scanning microscope (TPLSM) and its point spread functions. (a) General layout of our TPLSM. Fluorescence emission was detected in nondescanned mode by photomultiplier tubes (PMTs). For ratiometric JC-1 imaging, green and red components of JC-1 emission were separated spectrally and detected by the two detection channels. (b) To estimate the spatial resolution, the point spread function of our TPLSM was determined from the intensity profiles of subresolution $(100 \mathrm{~nm})$ beads. Displayed intensity profiles are the averages of 24 beads, and excitation wavelength was $800 \mathrm{~nm}$. Their full width at half maximum (FWHM) yields a lateral (X,Y) resolution of $0.4 \mu \mathrm{m}$ and an axial (Z) resolution of $1.4 \mu \mathrm{m}$. 

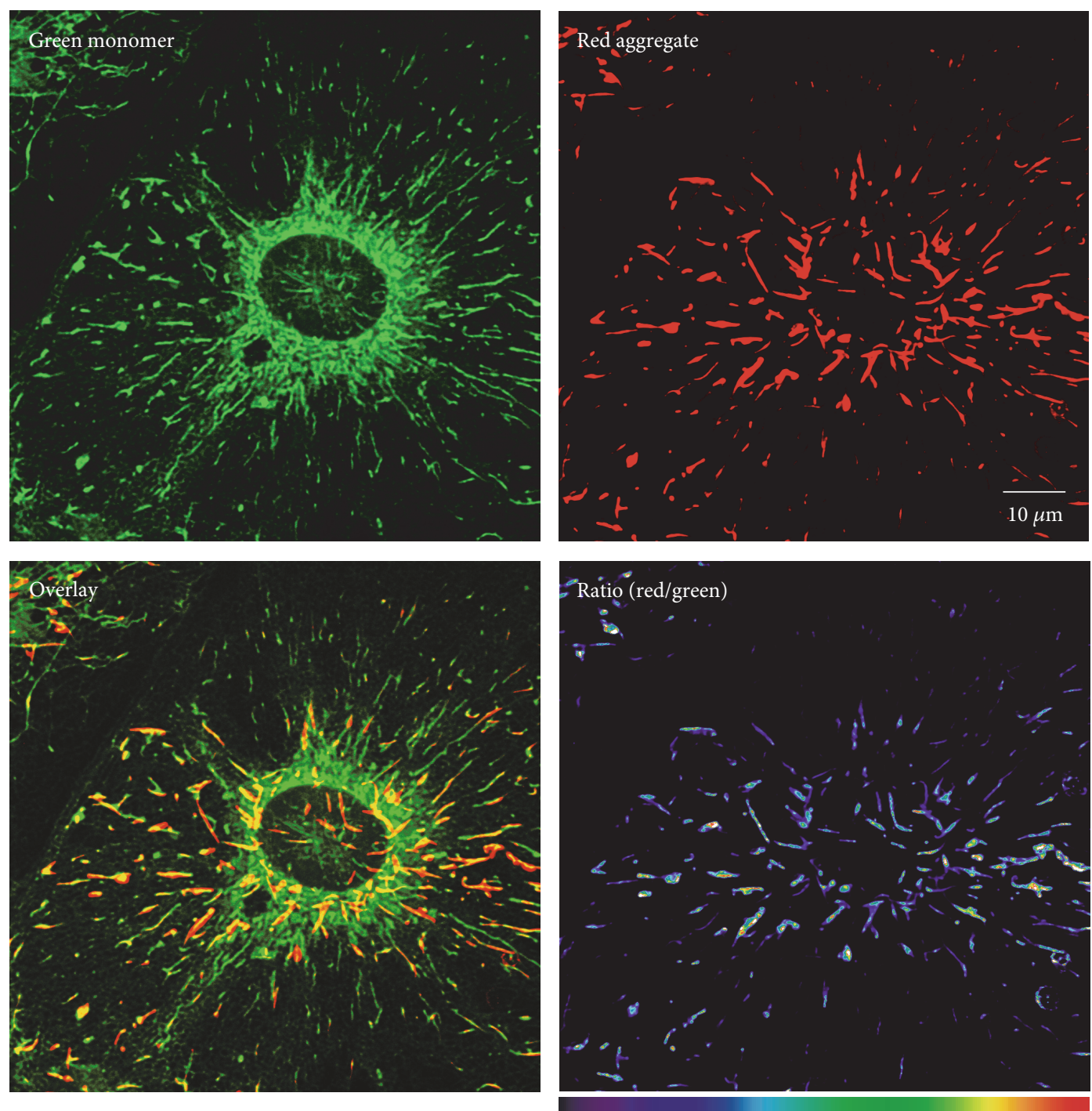

Depolarized

$\Delta \Psi \mathrm{m}$

Hyperpolarized

Figure 2: Sample images of a JC-1-labeled hippocampal astrocyte. Displayed are the individual raw images acquired by the two detection channels, representing JC-1 monomers and J-aggregates, respectively. The offline computed overlay image confirms the perfect alignment of the spectrally differing images. The calculated JC-1 ratio (red/green) represents the $\Delta \Psi \mathrm{m}$ range of individual mitochondria and their morphological/functional heterogeneity.

Student's $t$-tests. In the diagrams, genotype-related differences are indicated by asterisks $\left({ }^{* * *} p<0.001,{ }^{* *} p<0.01\right.$, and $\left.{ }^{*} p<0.05\right)$, and genotype-matched differences among recording conditions (drug-induced effects) are marked by crosshatches $\left({ }^{\# \#} p<0.001,{ }^{\# \#} p<0.01\right.$, and $\left.{ }^{\#} p<0.05\right)$.

\section{Results}

JC-1 is present as either monomer or J-aggregate. The monomers predominate in depolarized mitochondria and emit green fluorescence $(\sim 530 \mathrm{~nm})$. Oligomers (J-aggregates) only form in mitochondria with a $\Delta \Psi \mathrm{m}<-140 \mathrm{mV}$ and emit red fluorescence $(\sim 590 \mathrm{~nm})$ [50-53]. Accordingly, JC-1 is ratiometric by emission, and-as we have shown earlier [54] - the relative green and red components of JC-1 fluorescence allow to distinguish mitochondria with high and low $\Delta \Psi \mathrm{m}$ and to detect spontaneous and evoked $\Delta \Psi \mathrm{m}$ changes. For the current analyses, an updated and further improved version of our TPLSM $[54,62]$ was used (Figure 1(a)). Therefore, we first ran initial tests with standardized fluorescent beads, to determine the lateral and axial resolution of the upgraded system (Figure 1(b)).

To analyze mitochondrial structure and function, cell cultures were bulk loaded with JC-1. Once labeled, astrocytes could be identified clearly by their large cell size and flatly grown shape (Figure 2). Individual astrocytes were then imaged as 3-d image stacks, selecting those cells whose boundaries could be identified clearly and which were not overlapping with other cells. Based on the acquired green and red channels, overlay and ratiometric images were then calculated offline (Figure 2). 

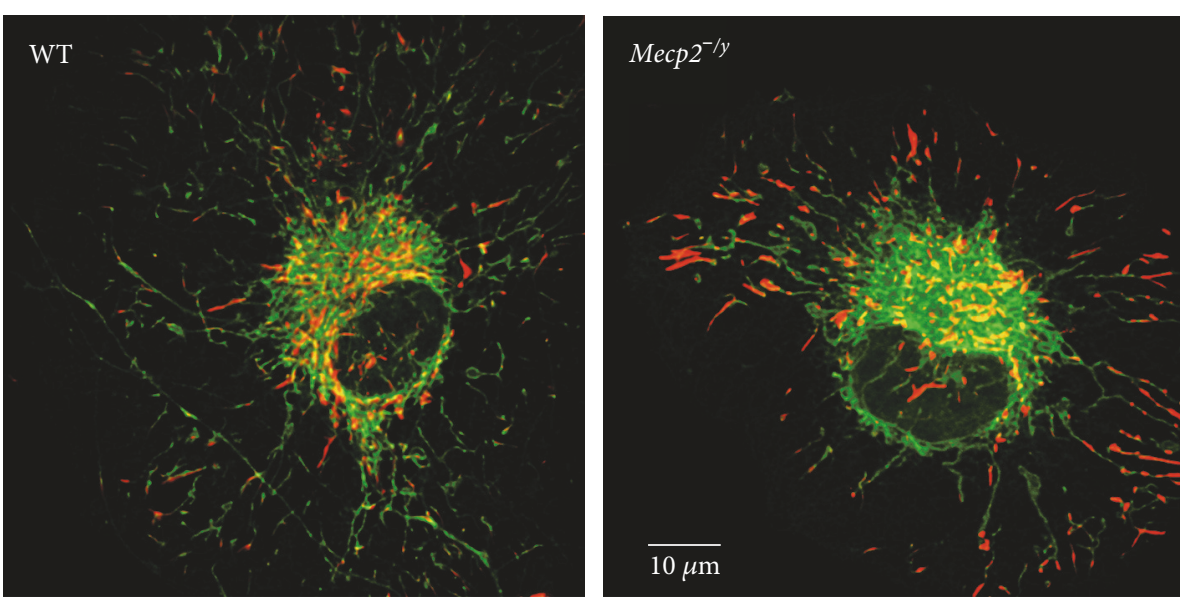

(a)

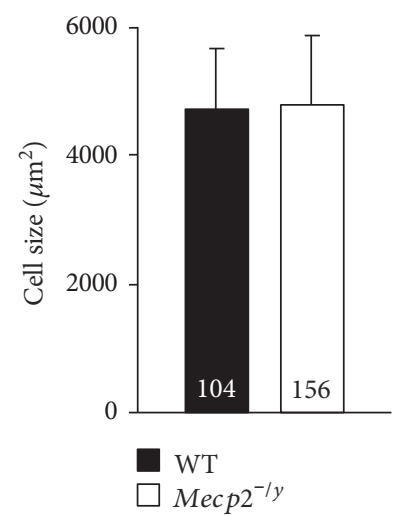

(b)

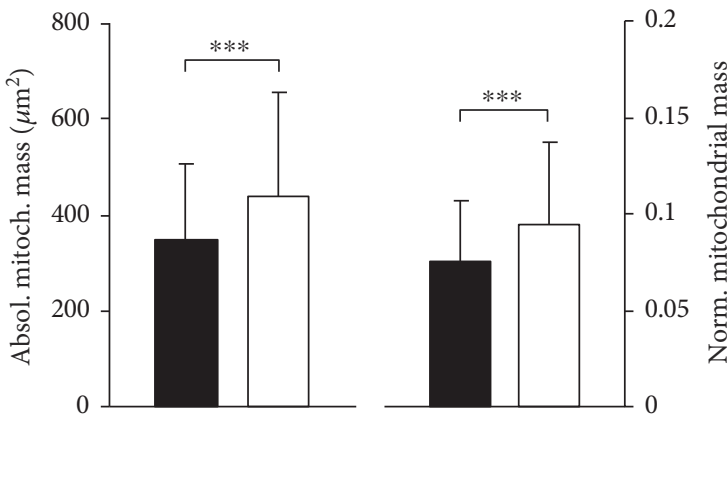

(c)

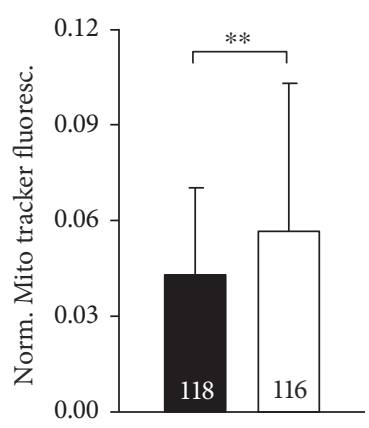

(d)

FIgURE 3: Increased mitochondrial mass in $M e c p 2^{-l y}$ astrocytes. (a) In general appearance, WT and Mecp $2^{-l y}$ astrocytes did not differ noticeably. Displayed images are overlays of the green and red JC-1 emissions. (b) Astrocytic cell size, determined by circling the outer cell boundaries, was also indistinguishable. Plotted data are mean \pm standard deviations, and the number of cells analyzed is included into each bar. Bar shading is identical for the following panels. (c) The absolute mitochondrial mass was higher in Mecp $2^{-l y}$ astrocytes than in WT cells (left side), and this difference was still present, when the mitochondrial mass was normalized to cell size (right side). Cell numbers analyzed are identical to panel B. Genotypic differences are indicated by asterisks $\left({ }^{* * *} p<0.001\right)$. (d) Using the mitochondria-specific marker, MitoTracker Red confirmed the increased mitochondrial mass in Mecp $2^{-l y}$ astrocytes. Displayed is the normalized intensity of astrocytic MitoTracker Red fluorescence as referred to individual cell size $\left({ }^{* *} p<0.01\right)$.

To identify potential differences in mitochondrial structure and/or function among WT and $M e c p 2^{-l y}$ astrocytes, these parameters had to be determined for individual mitochondria. Therefore, we developed a semiautomated routine to detect and analyze single particles. First, by blind deconvolution, pixel noise and out-of-focus light were removed, resulting in an improved contrast. Then, the best focal plane of the deconvolved stack was identified by visual inspection and collapsed with its adjacent two upper and two lower planes into a single plane, before thresholding was performed to obtain a binary mask discriminating among mitochondria and background. Spatial filtering then excluded all particles overlapping with others (ramified particles), lying at the margin of the image (truncated particles), or lying outside the size expected for mitochondria (individual bright pixels, or large aggregates in which individual organelles could not be discriminated). The resulting particle mask then included only those mitochondria, which would also be identified visually as individual particles. For each of these particles, the morphological characteristics (particle length) and the functional information (JC-1 ratio) were then extracted from the ratiometric source image.

In total, $104 \mathrm{WT}$ and $156 \mathrm{Mecp}^{-/ y}$ astrocytes were imaged and underwent the abovementioned analysis routines. Furthermore, we determined the size of each cell-by circling the outer cell boundaries-as well as the total volume of all JC-1-labeled structures. As total brain size and neuronal complexity differ among WT and Rett mice $[42,63]$, we first assessed potential genotypic differences in the size of the hippocampal astrocytes. With average cell areas of 4702 $\pm 950 \mu \mathrm{m}^{2}(n=104)$ and $4774 \pm 1097 \mu \mathrm{m}^{2}(n=156)$, respectively, cultured WT and Mecp $2^{-l y}$ astrocytes did not differ at all (Figures 3(a) and 3(b)). Yet, total mitochondrial mass was higher in $M e c p 2^{-l y}\left(440 \pm 214 \mu \mathrm{m}^{2}\right)$ than in WT astrocytes $\left(348 \pm 160 \mu \mathrm{m}^{2}\right)$ (Figure 3(c)). This still was the case, when mitochondrial content was normalized to cell size, 

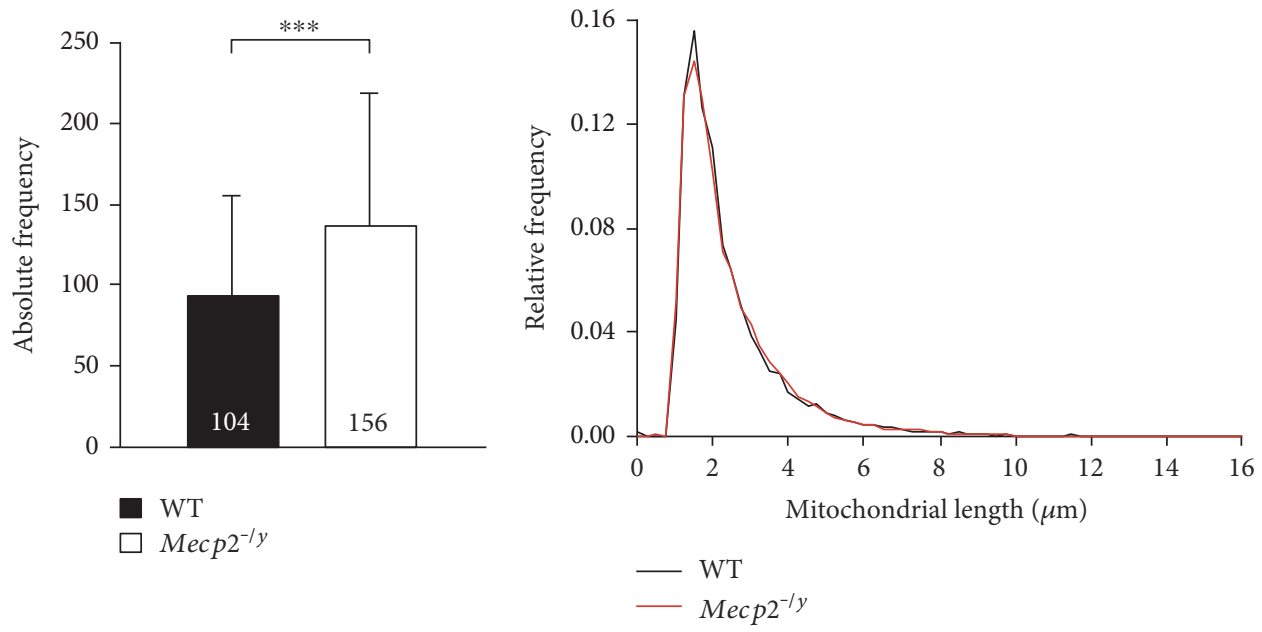

(a)

(b)

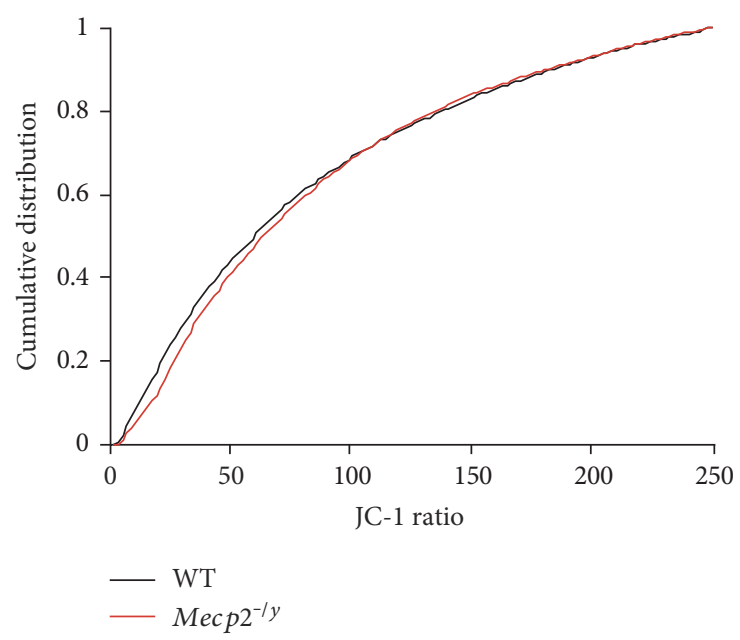

(c)

FIGURE 4: Functional parameters of individual mitochondria. (a) In line with the increased total mitochondrial mass in $M e c p 2^{-l y}$ astrocytes, also more individual organelles could be detected in these cells as compared to WT $\left.{ }^{* * *} p<0.001\right)$. (b) Genotypic differences in the length of the individual mitochondria were not found. The histogram-type distribution represents the entity of all individual mitochondrial particles detected by the automated analyses in WT (9769 mitochondria) and Mecp $2^{-l y}$ astrocytes (21,424 mitochondria). (c) The degree of polarization did not differ either among the genotypes. Plotted is the cumulative distribution function of the $\Delta \Psi \mathrm{m}$ of individual mitochondria of WT and Mecp $2^{-l y}$ astrocytes. It is based on the distribution of all recorded JC-1 ratios and indicates on the ordinate the probability that a WT or Mecp $2^{-l y}$ mitochondrion has a given $\Delta \Psi \mathrm{m}$ (or less).

yielding an average mitochondrial density of $0.094 \pm 0.044 /$ $\mu \mathrm{m}^{2}$ for $M e c p 2^{-l y}$ astrocytes $(n=156)$ and $0.076 \pm 0.032$ / $\mu \mathrm{m}^{2}$ for WT astrocytes $(n=104$, Figure $3(\mathrm{c}))$.

To validate this finding, we ran control experiments with the established mitochondrial marker MitoTracker Red [53, 64]. Images of individual astrocytes were acquired with a CCD-camera system, and the cellular intensity of MitoTracker Red fluorescence was normalized to the size of the respective cell. Again, the more intense fluorescence detected in $M e c p 2^{-l y}(0.057 \pm 0.046 ; n=116)$ than in WT astrocytes $(0.043 \pm 0.028 ; n=118)$ indicated a higher mitochondrial content for $M e c p 2^{-l y}$ astrocytes (Figure 3(d)).

To take a closer look at the genotypic difference in mitochondrial mass, we performed single particle analyses on JC1-labeled mitochondria. A total of 9769 mitochondria in WT and 21,424 mitochondria in $M e c p 2^{-/ y}$ astrocytes were classified, and more individual mitochondria could be detected in Mecp $2^{-l y}(136.5 \pm 81.5 /$ cell, $n=156)$ than in WT astrocytes (93.1 $\pm 6.0 /$ cell, $n=104)$ (Figure $4(\mathrm{a})$ ). Comparing the morphological characteristics, that is, the length of individual mitochondria, did however not reveal any genotypic differences. On average, mitochondria measured $2.34 \mu \mathrm{m}$ in WT and $3.32 \mu \mathrm{m}$ in $\mathrm{Mecp}^{-/ y}$ astrocytes (Figure 4(b)). Analyzing the average degree of polarization, assessed as JC-1 ratio, did not reveal any differences either, as indicated by the cumulative distribution function of their $\Delta \Psi \mathrm{m}$ (Figure 4(c)). In addition, correlation analysis confirmed that the size of a given mitochondrion does not correlate with its $\Delta \Psi \mathrm{m}$ (WT correlation coefficient 0.0895 ; $\mathrm{Mecp}^{-/ y}$ correlation coefficient 0.103$)$. 

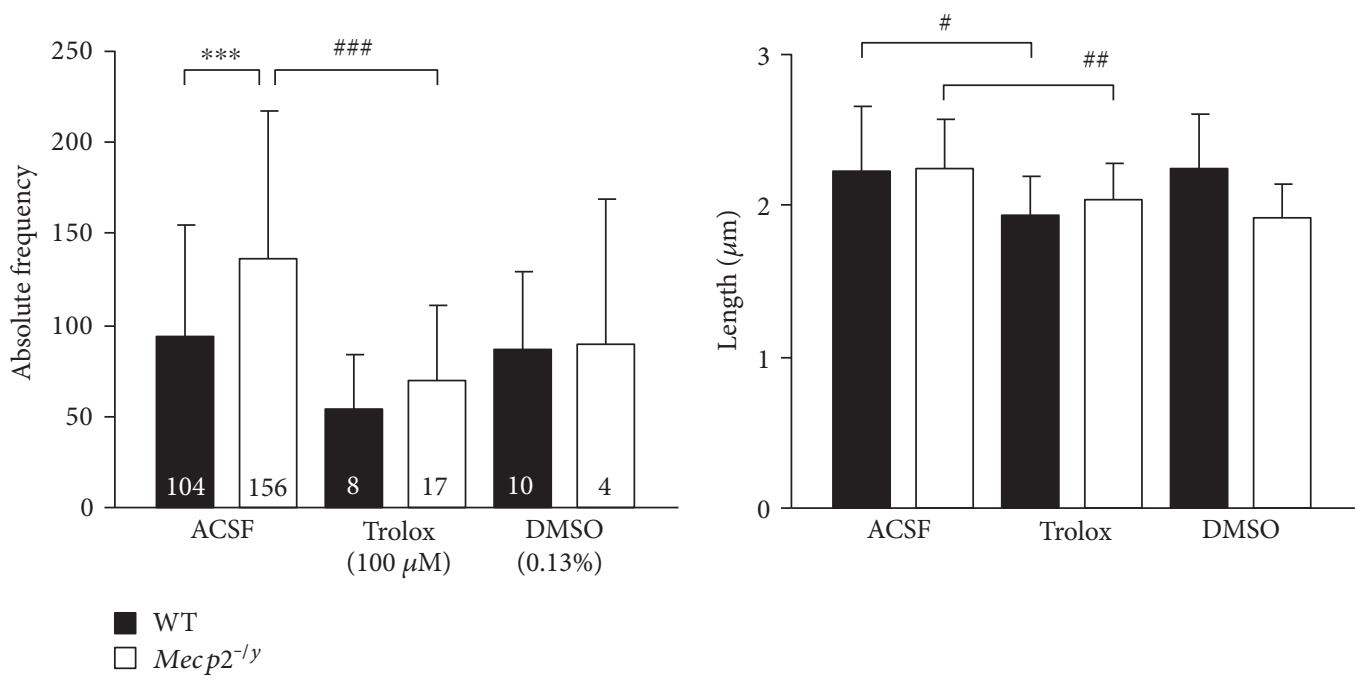

(a)

(b)

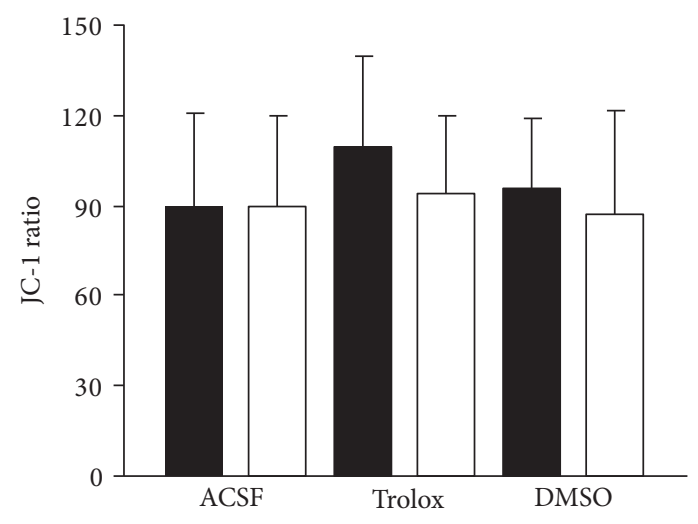

(c)

FIGURE 5: Free-radical scavenger treatment abolishes the differences in mitochondrial mass between WT and Mecp2 $2^{-l y}$ astrocytes. (a) Overnight Trolox treatment decreased (or tended to decrease) mitochondrial mass. As a result, the genotypic difference seen under control conditions among WT and Mecp $2^{-l y}$ astrocytes was no longer detectable. The solvent DMSO itself also tended to decrease the mitochondrial content of $M e c p 2^{-l y}$ astrocytes, but not to the degree seen with Trolox. Bar shading and the number of cells analyzed apply also to the following two panels. Genotpye-related differences are indicated by asterisks $\left({ }^{* * *} p<0.001\right)$ and drug-induced genotypematched differences by crosshatches $\left.{ }^{\# \# \#} p<0.001\right)$. (b) Trolox slightly but significantly decreased the length of individual mitochondria to an equal degree in WT and Mecp $2^{-l y}$ astrocytes. $\left({ }^{\#} p<0.05,{ }^{\# \#} p<0.01\right)$. (c) Significant changes in $\Delta \Psi \mathrm{m}$ could not be observed in response to Trolox treatment. In WT astrocytes, a trend towards increased $\Delta \Psi \mathrm{m}$ became obvious though.

\subsection{Modulation of Mitochondrial Parameters by Radical} Scavenger Treatment. Lowered vitamin E levels were found in the blood plasma of Rett patients [65]. Also, we previously confirmed that the vitamin E derivative Trolox improves cellular redox balance as well as synaptic function of the $M e c p 2^{-l y}$ hippocampus in vitro $[40,66]$, and it ameliorates some RTT symptoms when applied systemically to $M \operatorname{cep} 2^{-l y}$ mice [67]. Therefore, we assessed potential Trolox-mediated effects on mitochondrial function by incubating cell cultures overnight with this free-radical scavenger $(100 \mu \mathrm{M}, 12-14 \mathrm{~h})$.

Interestingly, Trolox significantly decreased the number of mitochondrial particles in $M e c p 2^{-l y}$ astrocytes and showed a corresponding trend $(p=0.076)$ in WT astrocytes (Figure 5(a)). As a result, the genotypic differences in mitochondrial density among WT and $M e c p 2^{-1 y}$ astrocytes were abolished (Figure 5(a)). Part of this effect can also be achieved by the solvent DMSO itself, which also mediates antioxidant capacity by scavenging hydroxyl radicals [68]. Furthermore, in both genotypes, the size of individual mitochondria was slightly smaller in Trolox-incubated than in untreated control cells (Figure 5(b)). Significant effects of Trolox on the $\Delta \Psi \mathrm{m}$ of WT or Mecp $2^{-l y}$ astrocytes were not found, but in WT astrocytes, a trend ( $p=0.093)$ towards increased (more negative) $\Delta \Psi \mathrm{m}$ became apparent (Figure 5(c)).

3.2. Cytosolic Redox Balance. To confirm the assumption that the differences in mitochondrial mass are closely associated with differences in redox balance among WT and $M e c p 2^{-1 y}$ astrocytes, we quantified cellular redox conditions by using the genetically encoded redox sensor roGFP1 (Figure 6(a)). Expressing roGFP1 in the cytosol reported for steady-state 


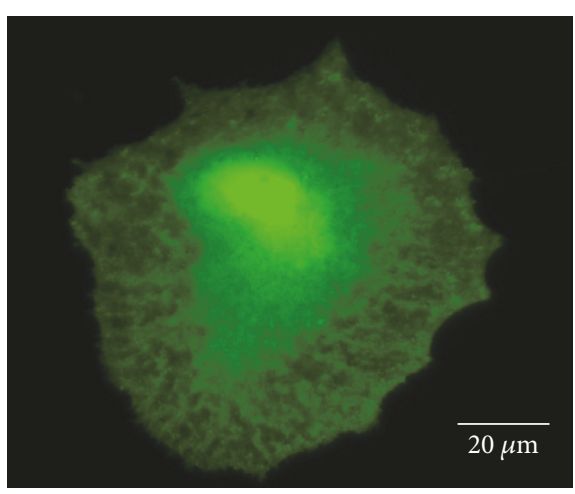

(a)

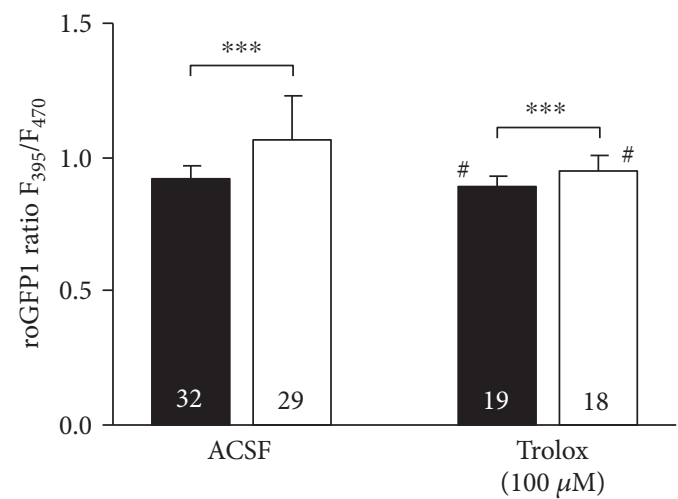

WT

$\operatorname{Mecp} 2^{-/ y}$

(b)
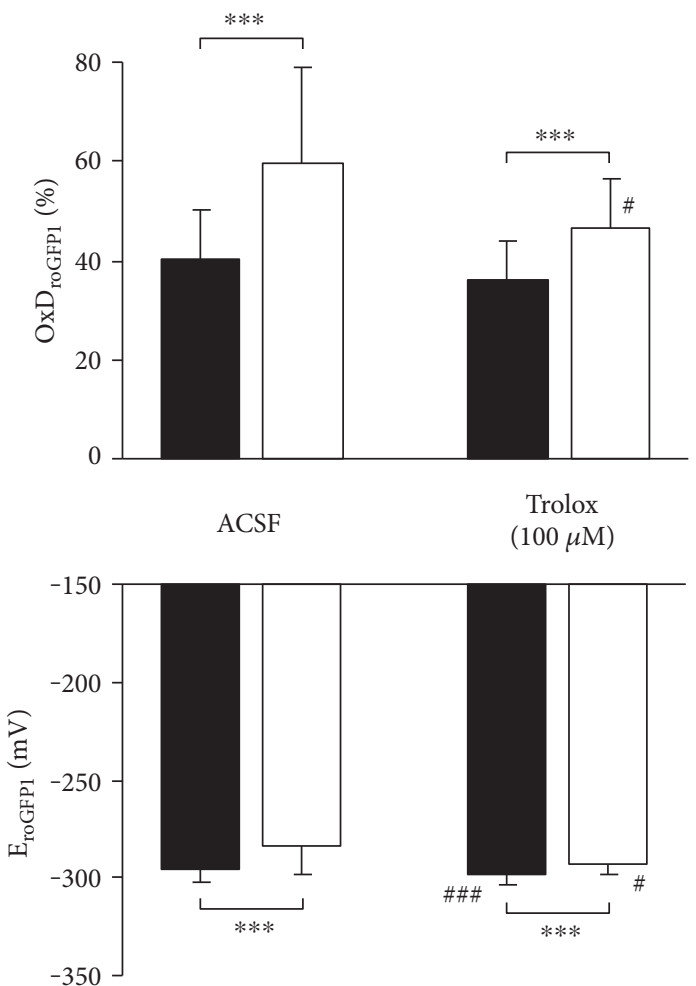

(c)

Figure 6: Optical redox imaging with the genetically encoded roGFP1 sensor confirms more oxidized conditions in Mecp $2^{-l y}$ astrocytes. (a) CCD camera image of a Mecp $2^{-l y}$ astrocyte expressing cytosolic roGFP1. (b) Under control conditions, fluorescence ratios were higher (more oxidized) in the cytosol of $M e c p 2^{-l y}$ than of WT astrocytes. Trolox induced a shift towards more reducing conditions especially in $M e c p 2^{-l y}$ astrocytes $\left({ }^{* * *} p<0.001,{ }^{*} p<0.05\right)$. (c) Calculating the relative degree of roGFP1 oxidation $\left(\mathrm{OxD}_{\mathrm{roGFP} 1}\right)$ and the corresponding reduction potentials $\left(\mathrm{E}_{\text {roGFP1 }}\right)$ confirms the more oxidized conditions in Mecp $2^{-l y}$ astrocytes as well as the antioxidant effect mediated by Trolox $\left(^{\# \# \#} p<0.001\right)$.

resting conditions a fluorescence ratio of $0.92 \pm 0.05(n=32)$ in WT astrocytes, whereas the fluorescence ratio in Mecp $2^{-l y}$ astrocytes was slightly higher, that is, more oxidized (1.06 $\pm 0.17, n=29$; Figure 6(b)). This also is evident from the relative degrees of roGFP1 oxidation (WT $40.4 \pm 9.8 \%$; Mecp $2^{-l y}$ $59.7 \pm 19.5 \%)$ as well as the corresponding reduction potentials (Figure 6(c)). To verify that Trolox $(100 \mu \mathrm{M})$ improved cellular redox balance, we also quantified cytosolic redox conditions after overnight treatment with this free-radical scavenger. Indeed in WT cells, the relative degree of roGFP1 oxidation slightly decreased to $36.0 \pm 7.7 \%(n=19)$, whereas it decreased more intensely in $M e c p 2^{-l y}$ astrocytes to 46.7 $\pm 9.9 \%(n=18)$. As a result of this treatment, the genotypic differences in roGFP1 ratio, relative roGFP1 oxidation level, and reduction potential became less pronounced as compared to the untreated astrocytes (Figures 6(b) and 6(c)). 

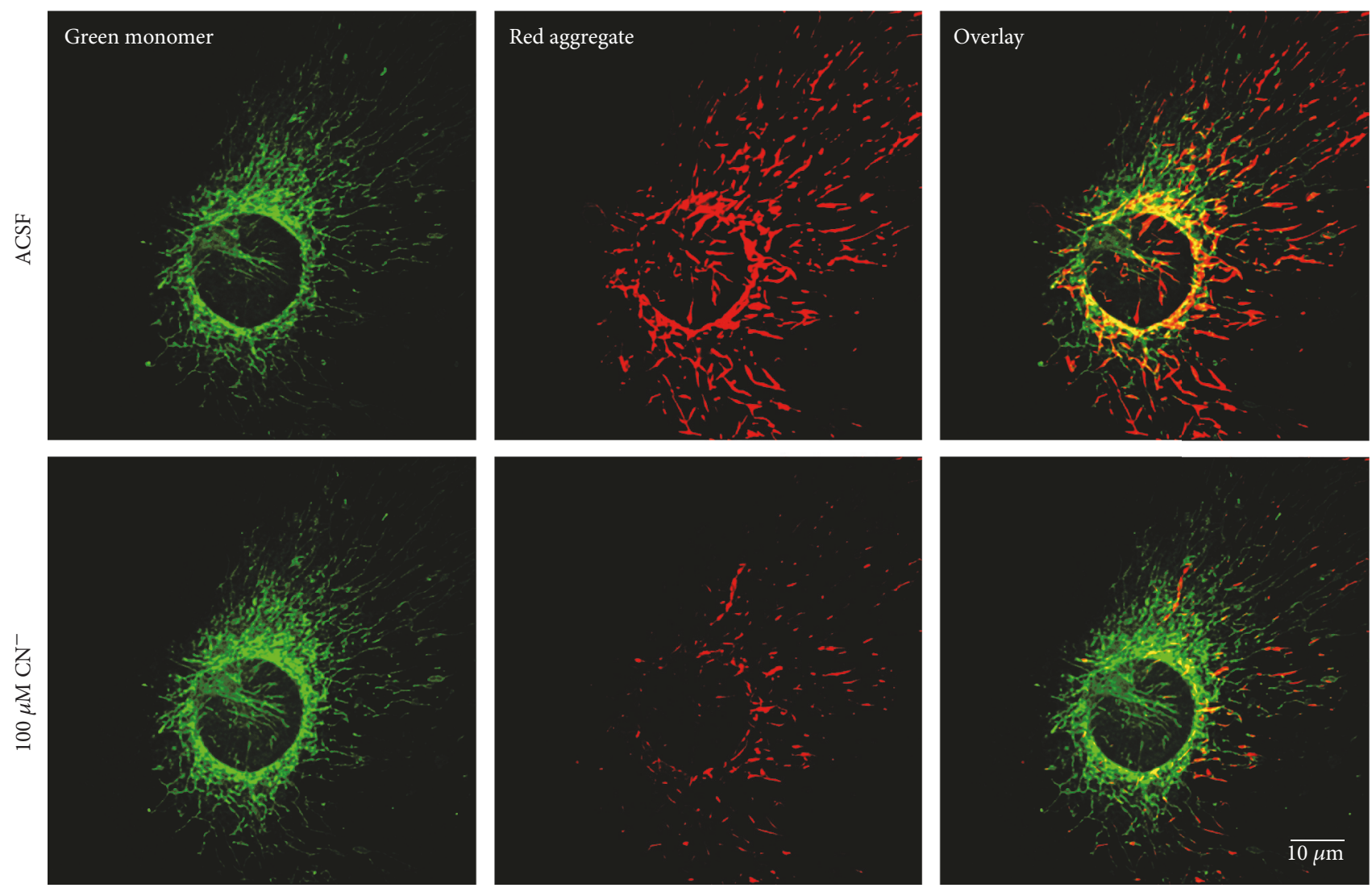

(a)
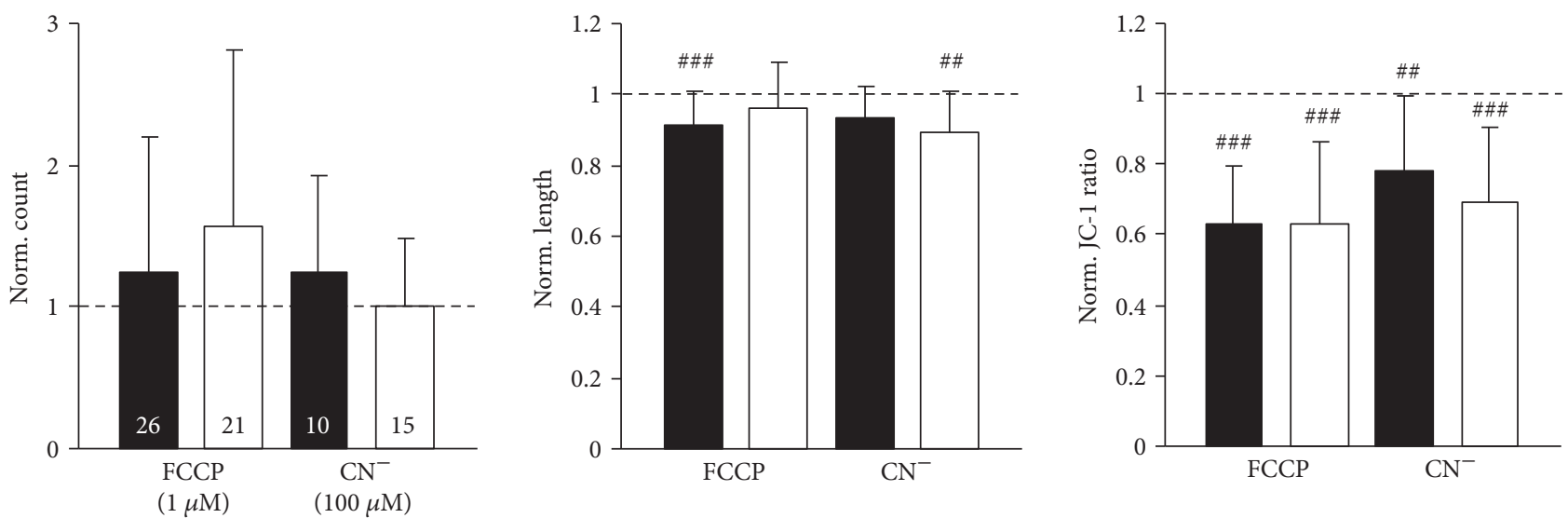

WT

$\operatorname{Mec} p 2^{-/ y}$

(b)

(c)

(d)

Figure 7: WT and Mecp2 $2^{-l y}$ astrocytes show similar vulnerabilities to mitochondrial compromise. (a) $\mathrm{CN}^{-}$treatment (100 $\left.\mu \mathrm{m}, 5 \mathrm{~min}\right)$ evoked a clear decrease in JC-1 ratio, which is evident as the green shift of the dual color emission and indicates a marked mitochondrial depolarization; displayed is a WT astrocyte. (b) Significant changes in mitochondrial content (mitochondria/cell) were not evoked by FCCP or $\mathrm{CN}^{-}$treatment. (c) The length of individual mitochondria was affected only very moderately $\left({ }^{\# \#} p<0.01,{ }^{\# \# \#} p<0.001\right)$. (d) As expected, both treatments evoked a clear mitochondrial depolarization. Significant genotypic differences were not observed in response to chemically induced hypoxia $\left(\mathrm{CN}^{-}\right)$or mitochondrial uncoupling (FCCP).

3.3. Effects of Mitochondrial Stressors. Previously, we found that the hippocampus of adult Mecp $2^{-l y}$ mice shows an exaggerated hypoxia susceptibility [69] and also in neonatal hippocampal organotypic slice cultures, we detected exaggerated redox responses to $\mathrm{O}_{2}$ shortage [40]. Therefore, we also assessed the effects of mitochondrial stress, by exposing cultured astrocytes to the mitochondrial uncoupler FCCP or by blocking mitochondrial respiration by $\mathrm{CN}^{-}$(Figure 7). To assess the effects of these compounds, each astrocyte was recorded before and after drug treatment, and all drug- 
induced changes were referred (normalized) to pretreatment control conditions.

Treatment with either FCCP $(1 \mu \mathrm{M}, 5 \mathrm{~min})$ or $\mathrm{CN}^{-}$ $(100 \mu \mathrm{M}, 5 \mathrm{~min})$ markedly decreased the JC-1 fluorescence ratio, indicating a pronounced mitochondrial depolarization (Figures 7(a) and 7(d)). A typical response of mitochondria exposed to such stress is the so-called thread-grain transition, that is, a disruption of longer mitochondrial filaments and a rounding of the individual particles [70-72]. Also here, this was elicited by FCCP in some astrocytes, and it resulted in a markedly increased variability of mitochondrial counts (Figure 7(b)) as well as in a moderately reduced length of the individual mitochondria (Figure 7(c)). As expected, the extent of the FCCP-induced mitochondrial depolarization was identical in WT and Mecp2 $2^{-l y}$ cells (Figure 7(d)); this compound acts as protonophore and completely collapses $\Delta \Psi \mathrm{m}$. Also $\mathrm{CN}^{-}$treatment elicited a pronounced mitochondrial depolarization in both genotypes, which tended to be slightly more intense in $M e c p 2^{-l y}$ than in WT astrocytes (Figure 7(d)). Nevertheless, obvious genotypic differences in mitochondrial vulnerability to these pharmacological insults were not detected.

\section{Discussion}

Due to the various alterations of mitochondrial structure and function, which are associated with RTT, mitochondrial dysfunction has been proposed to contribute to disease progression (see [18-20, 28, 29]). Earlier, we found in the hippocampal slices of neonatal Mecp $2^{-l y}$ mice an increased ratio of FAD/NADH autofluorescence, which indicates an intensified mitochondrial metabolism [40]. This difference was present in st. pyramidale, which is largely dominated by pyramidal cell somata, but also in st. radiatum, a mixed layer containing neurons and glia. Hence, glial cells may show mitochondrial alterations as well. Therefore, we now conducted optical analyses in WT and $M e c p 2^{-l y}$ glia to characterize further the spectrum of mitochondrial alterations in RTT. Due to their pivotal roles in extracellular ion homeostasis [73], transmitter uptake [74], and blood-brain barrier formation [75], we focused on astrocytes.

The earliest analyses of mitochondria in RTT took advantage of electron microscopy to assess changes in the mitochondrial ultrastructure $[20,22,76]$, but this approach lacks functional information. Later, biochemical and molecular biological assays were performed to identify altered enzyme activities and protein levels [23, 25, 29, 77]. Such assays are usually based on full brain or large tissue samples and yield valuable functional insights, but they do not provide single cell resolution. Our 2-photon microscopy study ensured both, functional information as well as subcellular resolution.

Using this technological advantage in combination with the developed semiautomated analysis-routines, we confirmed that mitochondria are more numerous in $M e c p 2^{-l y}$ hippocampal astrocytes. Since the individual mitochondria did not differ in their size and since genotypic differences in cell dimensions were not found, this points out to an increased total mitochondrial mass in $M e c p 2^{-l y}$ astrocytes.
One may argue now that the higher number of individual mitochondria identified in cultured $M e c p 2^{-l y}$ astrocytes could arise from a less dense mitochondrial packaging, which facilitated a successful single-particle detection in these cells. Yet, the total mitochondrial content determined in parallel and labeling with the mitochondria-specific marker MitoTracker Red also confirm an increased mitochondrial mass in $M e c p 2^{-l y}$ astrocytes. It therefore seems that the increased mitochondrial content constitutes a cellendogenous response to compensate for the mitochondriarelated deficits and their limited metabolic/respiratory capacity in RTT $[26,27,31,40]$. Interestingly, an increased mitochondrial mass was also reported for $M e c p 2^{-l y}$ microglia of the juvenile mouse brain [78]. In contrast, Mecp2-null mouse skin fibroblasts (cultured in regular medium) and stem-cell derived Mecp2-mutant neurons do not differ in their mitochondrial contents $[79,80]$.

Obvious differences in the size or $\Delta \Psi \mathrm{m}$ of individual mitochondria could not be detected among WT and $M e c p 2^{-l y}$ astrocytes. This suggests that the immediate functional impact of the mitochondrial alterations in RTT is rather subtle, which is also indicated by the fact that-in contrast to other mitochondriopathies-a marked degree of neurodegeneration is not evident in RTT [41].

Earlier studies have convincingly linked RTT to cellular redox changes and oxidative stress [37, 40,81]. The conducted redox analyses now confirm for the first time that the oxidative burden in RTT also applies to astrocytes. As we verified earlier, the free-radical scavenger Trolox improved the cellular redox balance in organotypic hippocampal slices [40], dampened neuronal hyperexcitability in adult hippocampal slices of symptomatic $M e c p 2^{-l y}$ mice, and also improved the hypoxia tolerance as well as certain types of synaptic plasticity [66, 67]. In these studies, any adverse impact of Trolox on mitochondrial function could be ruled out, and also here, an overnight incubation of astrocytes with Trolox did not affect mitochondrial shape or $\Delta \Psi \mathrm{m}$. However, Trolox incubation clearly improved cytosolic redox balance in $\mathrm{WT}$ and even more so in $M e c p 2^{-l y}$ astrocytes, thereby dampening the genotypic differences and opposing the oxidative stress in Mecp $2^{-l y}$ cells. As Trolox also slightly reduced the number of mitochondria per cell and thereby eliminated the genotypic differences seen in untreated astrocytes, it can therefore be concluded that the redox imbalance in RTT is one of the factors that underlies the increased mitochondrial mass in Mecp $2^{-l y}$ astrocytes.

Furthermore, we assessed potentially different responses of WT and $M e c p 2^{-l y}$ astrocytes to different mitochondriadirected drugs. FCCP evoked marked mitochondrial depolarizations in both genotypes by abolishing the proton gradient across the inner mitochondrial membrane. In addition, it slightly reduced the size of mitochondria in WT astrocytes. As structural changes, or even mitochondrial fragmentation, are quite commonly induced by FCCP [70, 82]; this explains the decreased mitochondrial length and increased number of individual mitochondria detected in some of the WT and $M e c p 2^{-l y}$ astrocytes upon mitochondrial uncoupling. The inhibition of respiratory complex IV by $\mathrm{CN}^{-}$also markedly depolarized mitochondria; the trend towards a more 
intense depolarization of $M e c p 2^{-l y}$ astrocytes may reflect the increased hypoxia susceptibility we found earlier in the hippocampal and brainstem slices of $M e c p 2^{-l y}$ mice [69, 83-85]. Furthermore, mitochondria of Mecp2 ${ }^{-l y}$ astrocytes became slightly smaller upon $\mathrm{CN}^{-}$treatment. Changes in mitochondrial content were, however, not detectable in any genotype. Therefore, as pronounced genotype-related changes were not detected for mitochondrial size, mitochondrial content, and $\Delta \Psi \mathrm{m}$, it has to be assumed that mitochondria in WT and $M e c p 2^{-l y}$ astrocytes do not noticeably differ in their vulnerabilities to uncoupling and chemical hypoxia. In view of the earlier detected increased hypoxia susceptibility of hippocampal and brainstem networks of MeCP2-deficient mice $[69,85]$, this is an important finding. Yet, it also has to be considered that the pharmacological challenges applied in the present study were quite intense.

Our 2-photon analyses were based on the emission ratiometric $\Delta \Psi \mathrm{m}$ indicator JC- 1 . We developed and thoroughly tested this JC-1-based emission ratiometric 2-photon imaging approach earlier [54], and it allows not only to visualize individual mitochondria but also to compare their $\Delta \Psi \mathrm{m}$ and quantify any $\Delta \Psi \mathrm{m}$ changes. JC-1 clearly differs from other dyes, as it is the only emission ratiometric indicator detecting $\Delta \Psi \mathrm{m}$ changes with sufficient sensitivity. Often criticized is that JC-1 reacts more slowly than other mitochondrial markers, which do not form aggregates. Hence, the true kinetics of fast $\Delta \Psi \mathrm{m}$ changes might be underestimated $[54,86]$. However, as we did not aim to resolve temporally any $\Delta \Psi \mathrm{m}$ changes, but rather compared steady-state conditions or drug treatment endpoints, this should be of no concern. A true drawback of JC-1 is, however, that comparative $\Delta \Psi \mathrm{m}$ analyses in more intact preparations are hardly possible, as the excessive background fluorescence of interstitial JC-1 monomers prevents a meaningful JC-1 staining in, for example, acute or organotypic tissue slices.

Nevertheless, JC-1 and its derivatives are the only ratiometric mitochondrial $\Delta \Psi \mathrm{m}$ indicators, and as such, their fluorescence response is not affected by the extent of dye uptake and/or differences in cellular mitochondrial content. All of these apply, however, to other nonratiometric $\Delta \Psi \mathrm{m}$ indicators (e.g., rhodamine 123 or TMRM), and it may markedly complicate data analyses, especially when these compounds are used in low-resolution approaches, such as flow-cytometry and cuvette-based spectrophotometric assays. Only recently, an improved variant of JC-1, termed JC-10, became available. It functions just as JC-1 but offers higher water solubility and an improved dynamic response range (see http://www .enzolifesciences.com/fileadmin/reports/Datasheet-ENZ-52305 .pdf). Thus, it may prove more advantageous also for future emission-ratiometric 2-photon imaging applications of $\Delta \Psi \mathrm{m}$ alterations in individual mitochondria or $\Delta \Psi \mathrm{m}$ differences among various cell types and/or genotypes.

\section{Conclusions}

Focusing on astrocytes, we performed functional optical analyses on the subcellular level, to extend earlier findings on mitochondrial alterations and redox imbalance in RTT. As the entire study is based on dissociated cell cultures, it can only reflect the neonatal developmental stage. Nevertheless, an increased mitochondrial mass and more oxidized cytosolic redox conditions were already detectable in $M e c p 2^{-l y}$ hippocampal astrocytes of presymptomatic mice. This genotypic difference in mitochondrial mass was obvious for absolute mitochondrial content, its normalization to cell size, and MitoTracker labeling. This confirms that also astrocytes undergo clear alterations already during the neonatal and presymptomatic stages of RTT, which further supports the hypothesis that mitochondrial alterations and the associated oxidative burden drive the progression of this neurodevelopmental disorder. Trolox did not mediate any adverse effects on mitochondria, which is certainly of interest for free-radical scavenger- and antioxidant-based pharmacotreatment concepts in RTT. More importantly, this free-radical scavenger successfully abolished the genotypic differences in mitochondrial content among WT and Mecp $2^{-l y}$ astrocytes and improved cytosolic redox balance especially in $M e c p 2^{-l y}$ astrocytes. This identifies cellular redox imbalance as one of the mechanisms underlying the increased mitochondrial mass in $M e c p 2^{-l y}$ astrocytes.

\section{Conflicts of Interest}

The authors declare that there is no conflict of interest regarding the publication of this paper.

\section{Acknowledgments}

The authors are grateful to Belinda Kempkes for her excellent technical assistance and to Professor S. James Remington (Institute of Molecular Biology, University of Oregon, Eugene, OR) for making the plasmids expressing roGFP1 available to them. This study was supported by the Cluster of Excellence and DFG Research Center Nanoscale Microscopy and Molecular Physiology of the Brain (CNMPB) as well as by the University Medical Center Göttingen and the State of Lower Saxony (Large-Scale Equipment Grant INST 1525/14-1 FUGG).

\section{References}

[1] A. Rett, "Über ein eigenartiges hirnatrophisches Syndrom bei Hyperammonämie im Kindesalter," Wiener Medizinische Wochenschrift (1946), vol. 116, pp. 723-726, 1966.

[2] B. Hagberg, J. Aicardi, K. Dias, and O. Ramos, "A progressive syndrome of autism, dementia, ataxia, and loss of purposeful hand use in girls: Rett's syndrome: report of 35 cases," Annals of Neurology, vol. 14, pp. 471-479, 1983.

[3] M. Chahrour and H. Y. Zoghbi, "The story of Rett syndrome: from clinic to neurobiology," Neuron, vol. 56, pp. 422-437, 2007.

[4] B. Hagberg, "Rett's syndrome: prevalence and impact on progressive severe mental retardation in girls," Acta Paediatrica Scandinavica, vol. 74, pp. 405-408, 1985.

[5] L. Villard, "MECP2 mutations in males," Journal of Medical Genetics, vol. 44, pp. 417-423, 2007. 
[6] R. E. Amir, I. B. VeyverVan den, M. Wan, C. Q. Tran, U. Francke, and H. Y. Zoghbi, "Rett syndrome is caused by mutations in X-linked MECP2, encoding methyl-CpGbinding protein 2," Nature Genetics, vol. 23, pp. 185-188, 1999.

[7] X. Nan, S. Cross, and A. Bird, "Gene silencing by methyl-CpGbinding proteins," Novartis Foundation Symposium, vol. 214, pp. 6-16, 1998, discussion 16-21, 46-50.

[8] M. Chahrour, S. Y. Jung, C. Shaw et al., "MeCP2, a key contributor to neurological disease, activates and represses transcription," Science, vol. 320, pp. 1224-1229, 2008.

[9] R. Trappe, F. Laccone, J. Cobilanschi et al., "MECP2 mutations in sporadic cases of Rett syndrome are almost exclusively of paternal origin," American Journal of Human Genetics, vol. 68, pp. 1093-1101, 2001.

[10] J. Dragich, I. Houwink-Manville, and C. Schanen, "Rett syndrome: a surprising result of mutation in MECP2," Human Molecular Genetics, vol. 9, pp. 2365-2375, 2000.

[11] B. Hagberg and I. Witt-Engerström, "Rett syndrome: a suggested staging system for describing impairment profile with increasing age towards adolescence," American Journal of Medical Genetics Supplement, vol. 1, pp. 47-59, 1986.

[12] B. Hagberg, M. Berg, and U. Steffenburg, "Three decades of sociomedical experiences from West Swedish Rett females 4-60 years of age," Brain dev, vol. 23, Supplement 1, pp. S28-S31, 2001.

[13] A. M. Kerr, D. D. Armstrong, R. J. Prescott, D. Doyle, and D. L. Kearney, "Rett syndrome: analysis of deaths in the British survey," European Child \& Adolescent Psychiatry, vol. 6, Supplement 1, pp. 71-74, 1997.

[14] C. A. Chapleau, J. Lane, L. Pozzo-Miller, and A. K. Percy, "Evaluation of current pharmacological treatment options in the management of Rett syndrome: from the present to future therapeutic alternatives," Current Clinical Pharmacology, vol. 8, pp. 358-369, 2013.

[15] D. M. Katz, A. Bird, M. Coenraads et al., "Rett syndrome: crossing the threshold to clinical translation," Trends in Neurosciences, vol. 39, pp. 100-113, 2016.

[16] M. Lotan and S. Hanks, "Physical therapy intervention for individuals with Rett syndrome," TheScientificWorldJOURNAL, vol. 6, pp. 1314-1338, 2006.

[17] S. B. Hanks, "The role of therapy in Rett syndrome," American Journal of Medical Genetics Supplement, vol. 1, pp. 247-252, 1986.

[18] M. Müller and K. Can, "Aberrant redox homoeostasis and mitochondrial dysfunction in Rett syndrome," Biochemical Society Transactions, vol. 42, pp. 959-964, 2014.

[19] N. Shulyakova, A. C. Andreazza, L. R. Mills, and J. H. Eubanks, "Mitochondrial dysfunction in the pathogenesis of Rett syndrome: implications for mitochondria-targeted therapies," Frontiers in Cellular Neuroscience, vol. 11, p. 58, 2017.

[20] O. Eeg-Olofsson, A. G. al-Zuhair, A. S. Teebi et al., "Rett syndrome: a mitochondrial disease?," Journal of Child Neurology, vol. 5, pp. 210-214, 1990.

[21] O. Eeg-Olofsson, A. G. Al-Zuhair, A. S. Teebi, and M. M. Al-Essa, "Abnormal mitochondria in the Rett syndrome," Brain and Development, vol. 10, pp. 260-262, 1988.

[22] M. E. Cornford, M. Philippart, B. Jacobs, A. B. Scheibel, and H. V. Vinters, "Neuropathology of Rett syndrome: case report with neuronal and mitochondrial abnormalities in the brain," Journal of Child Neurology, vol. 9, pp. 424-431, 1994.
[23] M. T. Dotti, L. Manneschi, A. Malandrini, N. StefanoDe, F. Caznerale, and A. Federico, "Mitochondrial dysfunction in Rett syndrome. An ultrastructural and biochemical study," Brain and Development, vol. 15, pp. 103-106, 1993.

[24] J. H. Gibson, B. Slobedman, K. N. Harikrishnan et al., "Downstream targets of methyl CpG binding protein 2 and their abnormal expression in the frontal cortex of the human Rett syndrome brain," BMC Neuroscience, vol. 11, no. 1 , p. 53, 2010.

[25] A. Pecorelli, G. Leoni, F. Cervellati et al., "Genes related to mitochondrial functions, protein degradation, and chromatin folding are differentially expressed in lymphomonocytes of Rett syndrome patients," Mediators of Inflammation, vol. 2013, Article ID 137629, 18 pages, 2013.

[26] B. FilippisDe, D. Valenti, L. Baride et al., "Mitochondrial free radical overproduction due to respiratory chain impairment in the brain of a mouse model of Rett syndrome: protective effect of CNF1," Free Radical Biology and Medicine, vol. 83, pp. 167-177, 2015.

[27] S. Kriaucionis, A. Paterson, J. Curtis, J. Guy, N. Macleod, and A. Bird, "Gene expression analysis exposes mitochondrial abnormalities in a mouse model of Rett syndrome," Molecular and Cellular Biology, vol. 26, pp. 5033-5042, 2006.

[28] D. Valenti, L. Baride, B. FilippisDe, A. Henrion-Caude, and R. A. Vacca, "Mitochondrial dysfunction as a central actor in intellectual disability-related diseases: an overview of Down syndrome, autism, Fragile X and Rett syndrome," Neuroscience and Biobehavioral Reviews, vol. 46, Part 2, pp. 202-217, 2014.

[29] W. A. Gold, S. L. Williamson, S. Kaur et al., "Mitochondrial dysfunction in the skeletal muscle of a mouse model of Rett syndrome (RTT): implications for the disease phenotype," Mitochondrion, vol. 15, pp. 10-17, 2014.

[30] G. Forlani, E. Giarda, U. Ala et al., "The MeCP2/YY1 interaction regulates ANT1 expression at 4q35: novel hints for Rett syndrome pathogenesis," Human Molecular Genetics, vol. 19, pp. 3114-3123, 2010.

[31] C. Cervellati, C. Sticozzi, A. Romani et al., "Impaired enzymatic defensive activity, mitochondrial dysfunction and proteasome activation are involved in RTT cell oxidative damage," Biochimica et Biophysica Acta, vol. 1852, pp. 2066-2074, 2015.

[32] T. Matsuishi, F. Urabe, A. K. Percy et al., "Abnormal carbohydrate metabolism in cerebrospinal fluid in Rett syndrome," Journal of Child Neurology, vol. 9, pp. 26-30, 1994.

[33] V. Saywell, A. Viola, S. Confort-Gouny, Y. FurLe, L. Villard, and P. J. Cozzone, "Brain magnetic resonance study of Mecp2 deletion effects on anatomy and metabolism," Biochemical and Biophysical Research Communications, vol. 340, pp. 776783, 2006.

[34] P. O. Julu, A. M. Kerr, F. Apartopoulos et al., "Characterisation of breathing and associated central autonomic dysfunction in the Rett disorder," Archives of Disease in Childhood, vol. 85, pp. 29-37, 2001.

[35] G. M. Stettner, S. Zanella, P. Huppke, J. Gärtner, G. Hilaire, and M. Dutschmann, "Spontaneous central apneas occur in the C57BL/6J mouse strain," Respiratory Physiology \& Neurobiology, vol. 160, pp. 21-27, 2008.

[36] D. M. Katz, M. Dutschmann, J. M. Ramirez, and G. Hilaire, "Breathing disorders in Rett syndrome: progressive neurochemical dysfunction in the respiratory network after 
birth," Respiratory Physiology \& Neurobiology, vol. 168, pp. 101-108, 2009.

[37] C. Sierra, M. A. Vilaseca, N. Brandi et al., "Oxidative stress in Rett syndrome," Brain and Development, vol. 23, Supplement 1, pp. S236-S239, 2001.

[38] C. FeliceDe, L. Ciccoli, S. Leoncini et al., "Systemic oxidative stress in classic Rett syndrome," Free Radical Biology and Medicine, vol. 47, pp. 440-448, 2009.

[39] C. FeliceDe, F. Della Ragione, C. Signorini et al., "Oxidative brain damage in Mecp2-mutant murine models of Rett syndrome," Neurobiology of Disease, vol. 68, pp. 66-77, 2014.

[40] E. Großer, U. Hirt, O. A. Janc et al., "Oxidative burden and mitochondrial dysfunction in a mouse model of Rett syndrome," Neurobiology of Disease, vol. 48, pp. 102-114, 2012.

[41] D. D. Armstrong, "Neuropathology of Rett syndrome," Journal of Child Neurology, vol. 20, pp. 747-753, 2005.

[42] P. V. Belichenko, E. E. Wright, N. P. Belichenko et al., "Widespread changes in dendritic and axonal morphology in Mecp2-mutant mouse models of Rett syndrome: evidence for disruption of neuronal networks," The Journal of Comparative Neurology, vol. 514, pp. 240-258, 2009.

[43] P. Moretti, J. M. Levenson, F. Battaglia et al., "Learning and memory and synaptic plasticity are impaired in a mouse model of Rett syndrome," The Journal of Neuroscience, vol. 26, pp. 319-327, 2006.

[44] N. Ballas, D. T. Lioy, C. Grunseich, and G. Mandel, "Non-cell autonomous influence of MeCP2-deficient glia on neuronal dendritic morphology," Nature Neuroscience, vol. 12, pp. 311-317, 2009.

[45] D. T. Lioy, S. K. Garg, C. E. Monaghan et al., "A role for glia in the progression of Rett's syndrome," Nature, vol. 475, pp. 497-500, 2011.

[46] M. V. Nguyen, C. A. Felice, F. Du et al., "Oligodendrocyte lineage cells contribute unique features to Rett syndrome neuropathology," The Journal of Neuroscience, vol. 33, pp. 18764-18774, 2013.

[47] I. Maezawa and L. W. Jin, "Rett syndrome microglia damage dendrites and synapses by the elevated release of glutamate," The Journal of Neuroscience, vol. 30, pp. 5346-5356, 2010.

[48] J. Guy, B. Hendrich, M. Holmes, J. E. Martin, and A. Bird, “A mouse Mecp2-null mutation causes neurological symptoms that mimic Rett syndrome," Nature Genetics, vol. 27, pp. 322-326, 2001.

[49] J. Weller, K. M. Kizina, K. Can, G. Bao, and M. Müller, "Response properties of the genetically encoded optical $\mathrm{H}_{2} \mathrm{O}_{2}$ sensor HyPer," Free Radical Biology and Medicine, vol. 76, pp. 227-241, 2014.

[50] T. J. Sick and M. A. Perez-Pinzon, "Optical methods for probing mitochondrial function in brain slices," Methods, vol. 18, pp. 104-108, 1999.

[51] S. T. Smiley, M. Reers, C. Mottola-Hartshorn et al., "Intracellular heterogeneity in mitochondrial membrane potentials revealed by a J-aggregate-forming lipophilic cation JC-1," Proceedings of the National Academy of Sciences of the United States of America, vol. 88, pp. 3671-3675, 1991.

[52] M. R. Duchen, A. Surin, and J. Jacobson, "Imaging mitochondrial function in intact cells," Methods in Enzymology, vol. 361, pp. 353-389, 2003.

[53] K. A. Foster, F. Galeffi, F. J. Gerich, D. A. Turner, and M. Müller, "Optical and pharmacological tools to investigate the role of mitochondria during oxidative stress and neurodegeneration," Progress in Neurobiology, vol. 79, pp. 136-171, 2006.

[54] V. C. Keil, F. Funke, A. Zeug, D. Schild, and M. Müller, "Ratiometric high-resolution imaging of JC-1 fluorescence reveals the subcellular heterogeneity of astrocytic mitochondria," Pflügers Archiv, vol. 462, pp. 693-708, 2011.

[55] M. Reers, S. T. Smiley, C. Mottola-Hartshorn, A. Chen, M. Lin, and L. B. Chen, "Mitochondrial membrane potential monitored by JC-1 dye," Methods in Enzymology, vol. 260, pp. 406-417, 1995.

[56] G. T. Hanson, R. Aggeler, D. Oglesbee et al., "Investigating mitochondrial redox potential with redox-sensitive green fluorescent protein indicators," The Journal of Biological Chemistry, vol. 279, pp. 13044-13053, 2004.

[57] F. Funke, F. J. Gerich, and M. Müller, "Dynamic, semiquantitative imaging of intracellular ROS levels and redox status in rat hippocampal neurons," NeuroImage, vol. 54, pp. 2590-2602, 2011.

[58] K. C. Wagener, B. Kolbrink, K. Dietrich et al., "Redox-indicator mice stably expressing genetically-encoded neuronal roGFP: versatile tools to decipher subcellular redox dynamics in neuropathophysiology," Antioxidants \& Redox Signaling, vol. 25, pp. 41-58, 2016.

[59] K. Can, S. Kügler, and M. Müller, "Live imaging of mitochondrial ROS production and dynamic redox balance in neurons," in Techniques to Investigate Mitochondrial Function in Neurons, S. Strack and Y. M. Usachev, Eds., pp. 179-197, Springer Science+Business Media, 2017.

[60] C. T. Dooley, T. M. Dore, G. T. Hanson, W. C. Jackson, S. J. Remington, and R. Y. Tsien, "Imaging dynamic redox changes in mammalian cells with green fluorescent protein indicators," The Journal of Biological Chemistry, vol. 279, pp. 22284-22293, 2004.

[61] A. J. Meyer and T. P. Dick, "Fluorescent protein-based redox probes," Antioxidants \& Redox Signaling, vol. 13, pp. 621-650, 2010.

[62] M. Müller, J. Schmidt, S. L. Mironov, and D. W. Richter, "Construction and performance of a custom-built two-photon laser scanning system," Journal of Physics D: Applied Physics, vol. 36, pp. 1747-1757, 2003.

[63] N. P. Belichenko, P. V. Belichenko, H. H. Li, W. C. Mobley, and U. Francke, "Comparative study of brain morphology in Mecp2 mutant mouse models of Rett syndrome," The Journal of Comparative Neurology, vol. 508, pp. 184-195, 2008.

[64] M. Poot, Y. Z. Zhang, J. A. Kramer et al., “Analysis of mitochondrial morphology and function with novel fixable fluorescent stains," The Journal of Histochemistry and Cytochemistry, vol. 44, pp. 1363-1372, 1996.

[65] P. Formichi, C. Battisti, M. T. Dotti, G. Hayek, M. Zappella, and A. Federico, "Vitamin E serum levels in Rett syndrome," Journal of the Neurological Sciences, vol. 156, pp. 227-230, 1998.

[66] O. A. Janc and M. Müller, “The free radical scavenger Trolox dampens neuronal hyperexcitability, reinstates synaptic plasticity, and improves hypoxia tolerance in a mouse model of Rett syndrome," Frontiers in Cellular Neuroscience, vol. 8, p. 56, 2014.

[67] O. A. Janc, M. A. Hüser, K. Dietrich et al., "Systemic radical scavenger treatment of a mouse model of Rett syndrome: merits and limitations of the vitamin E derivative Trolox," Frontiers in Cellular Neuroscience, vol. 10, p. 266, 2016. 
[68] J. W. Phillis, A. Y. Estevez, and M. H. O'Regan, "Protective effects of the free radical scavengers, dimethyl sulfoxide and ethanol, in cerebral ischemia in gerbils," Neuroscience Letters, vol. 244, pp. 109-111, 1998.

[69] M. Fischer, J. Reuter, F. J. Gerich et al., "Enhanced hypoxia susceptibility in hippocampal slices from a mouse model of Rett syndrome," Journal of Neurophysiology, vol. 101, pp. 1016-1032, 2009.

[70] M. Müller, S. L. Mironov, M. V. Ivannikov, J. Schmidt, and D. W. Richter, "Mitochondrial organization and motility probed by two-photon microscopy in cultured mouse brainstem neurons," Experimental Cell Research, vol. 303, pp. 114$127,2005$.

[71] G. L. Rintoul, A. J. Filiano, J. B. Brocard, G. J. Kress, and I. J. Reynolds, "Glutamate decreases mitochondrial size and movement in primary forebrain neurons," The Journal of Neuroscience, vol. 23, pp. 7881-7888, 2003.

[72] V. P. Skulachev, "Mitochondrial filaments and clusters as intracellular power-transmitting cables," Trends in Biochemical Sciences, vol. 26, pp. 23-29, 2001.

[73] E. A. Newman, D. A. Frambach, and L. L. Odette, "Control of extracellular potassium levels by retinal glial cell $\mathrm{K}^{+}$siphoning," Science, vol. 225, pp. 1174-1175, 1984.

[74] L. K. Bak, A. Schousboe, and H. S. Waagepetersen, "The glutamate/GABA-glutamine cycle: aspects of transport, neurotransmitter homeostasis and ammonia transfer," Journal of Neurochemistry, vol. 98, pp. 641-653, 2006.

[75] R. C. Janzer and M. C. Raff, "Astrocytes induce blood-brain barrier properties in endothelial cells," Nature, vol. 325, pp. 253-257, 1987.

[76] A. Ruch, T. W. Kurczynski, and M. E. Velasco, "Mitochondrial alterations in Rett syndrome," Pediatric Neurology, vol. 5, pp. 320-323, 1989.

[77] S. B. Coker and A. R. Melnyk, "Rett syndrome and mitochondrial enzyme deficiencies," Journal of Child Neurology, vol. 6, pp. 164-166, 1991.

[78] L. W. Jin, M. Horiuchi, H. Wulff et al., "Dysregulation of glutamine transporter SNAT1 in Rett syndrome microglia: a mechanism for mitochondrial dysfunction and neurotoxicity," The Journal of Neuroscience, vol. 35, pp. 2516-2529, 2015.

[79] Y. Li, H. Wang, J. Muffat et al., "Global transcriptional and translational repression in human-embryonic-stem-cellderived Rett syndrome neurons," Cell Stem Cell, vol. 13, pp. 446-458, 2013.

[80] N. O. Shulyakova, "Studies of mitochondrial dysfunction in models of Rett syndrome. Thesis," Department of Physiology, University of Toronto, Toronto, Canada, 2016.

[81] C. FeliceDe, C. Signorini, S. Leoncini et al., "The role of oxidative stress in Rett syndrome: an overview," Annals of the New York Academy of Sciences, vol. 1259, pp. 121-135, 2012.

[82] D. F. Suen, K. L. Norris, and R. J. Youle, "Mitochondrial dynamics and apoptosis," Genes \& Development, vol. 22, pp. 1577-1590, 2008.

[83] M. Kron and M. Müller, "Enhanced anoxia susceptibility of MeCP2-deficient CA1 neurons involves altered $\mathrm{K}^{+}$conductances," Society for Neuroscience, Abstract Viewer/Itinerary Planner, p. 732.736, 2009.
[84] M. Kron and M. Müller, "Impaired hippocampal $\mathrm{Ca}^{2+}$ homeostasis and concomitant $\mathrm{K}^{+}$channel dysfunction in a mouse model of Rett syndrome during anoxia," Neuroscience, vol. 171, pp. 300-315, 2010.

[85] M. Kron, J. L. Zimmermann, M. Dutschmann, F. Funke, and M. Müller, "Altered responses of MeCP2-deficient mouse brain stem to severe hypoxia," Journal of Neurophysiology, vol. 105, pp. 3067-3079, 2011.

[86] A. Mathur, Y. Hong, B. K. Kemp, A. A. Barrientos, and J. D. Erusalimsky, "Evaluation of fluorescent dyes for the detection of mitochondrial membrane potential changes in cultured cardiomyocytes," Cardiovascular Research, vol. 46, pp. 126$138,2000$. 


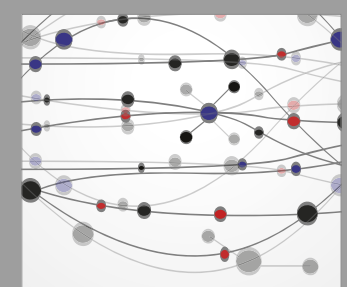

The Scientific World Journal
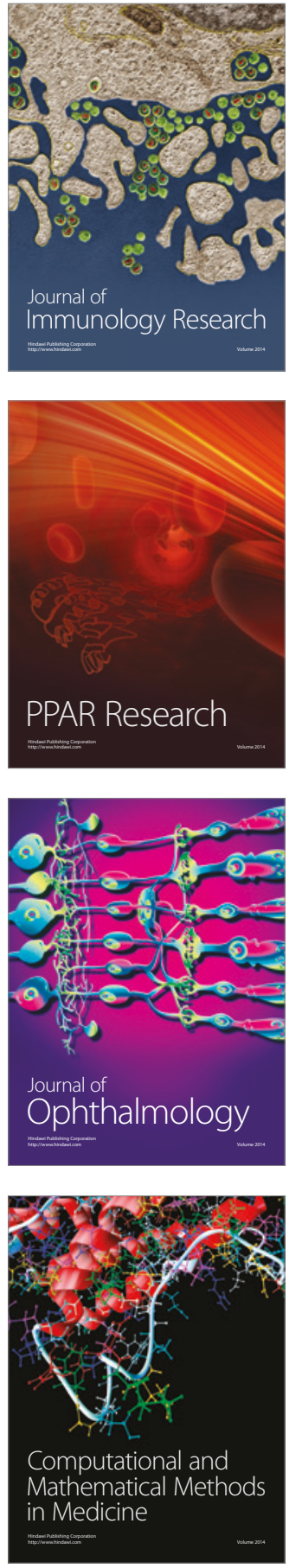

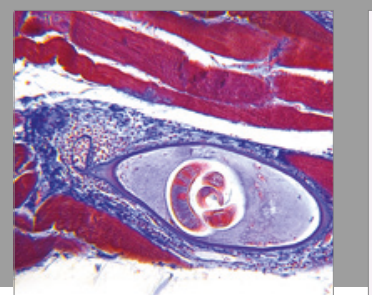

Gastroenterology Research and Practice
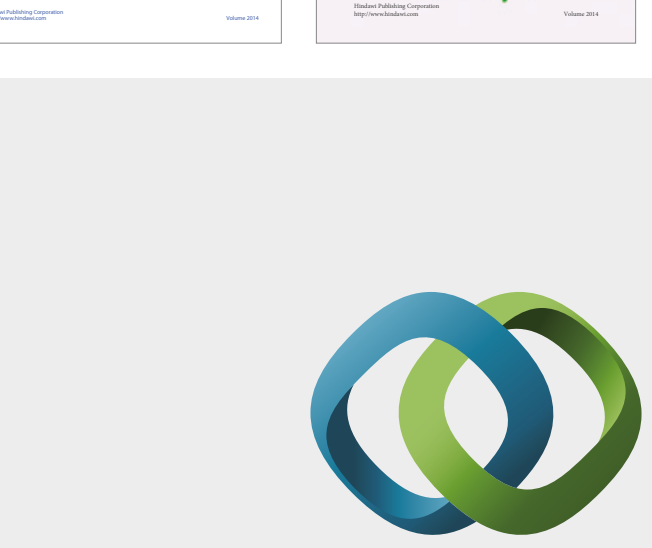

\section{Hindawi}

Submit your manuscripts at

https://www.hindawi.com
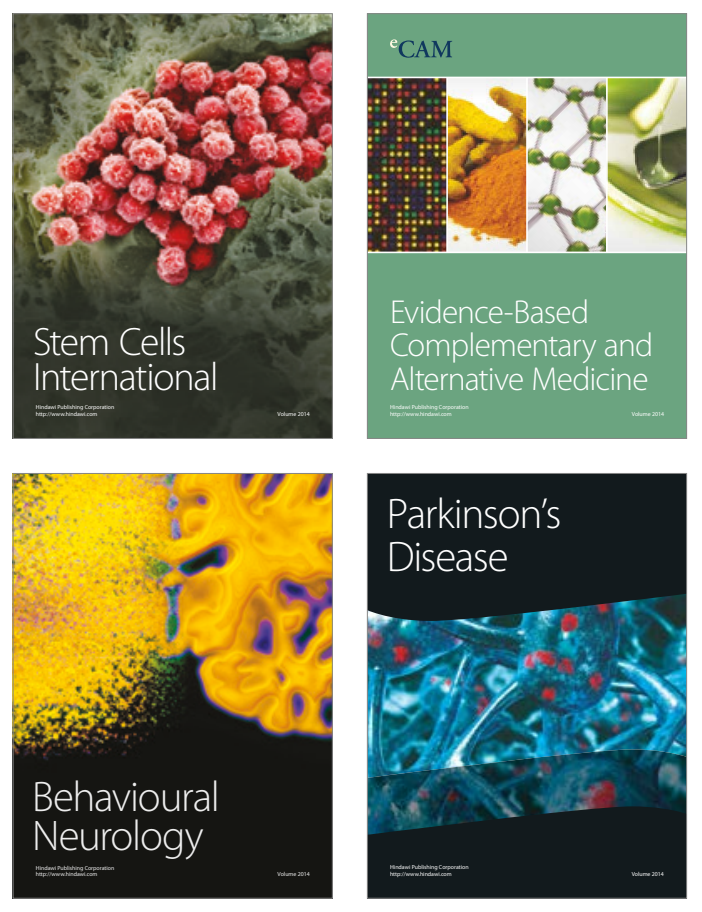
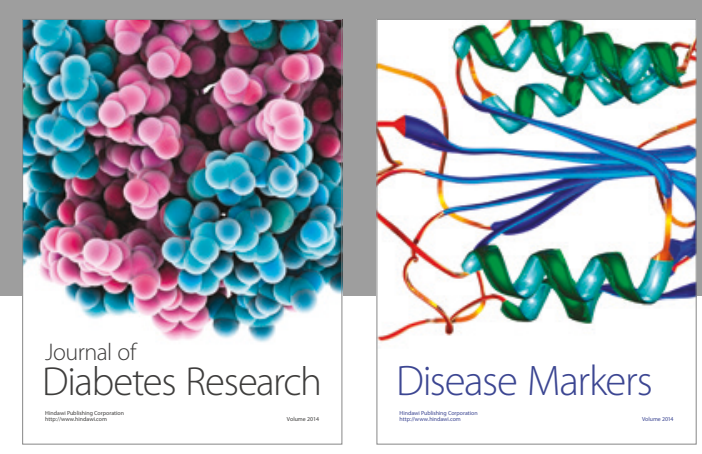

Disease Markers
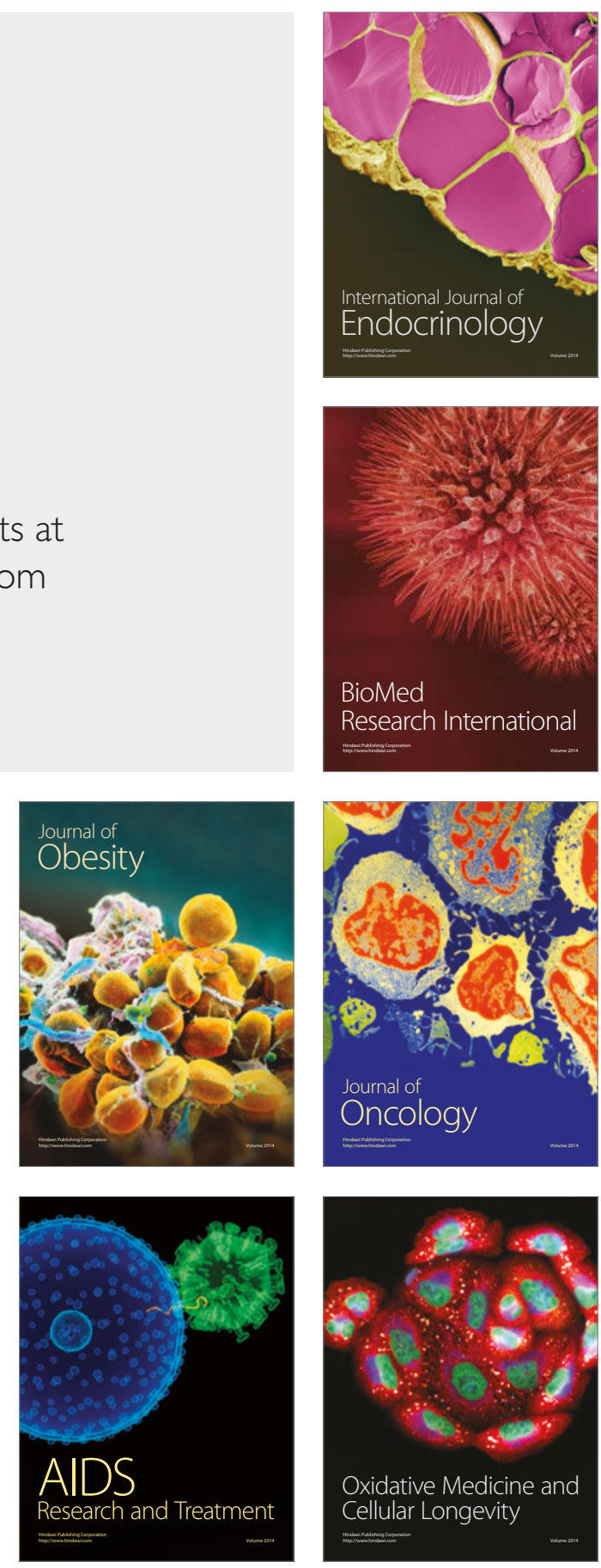